\title{
A NEW APPROACH TO NONLINEAR MODELLING OF DYNAMIC SYSTEMS BASED ON FUZZY RULES
}

\author{
ŁUKASZ BARTCZUK ${ }^{a}$, ANDRZEJ PRZYBYŁ ${ }^{a}, \operatorname{KRZYSZTOF~CPAŁKA~}^{a, *}$ \\ ${ }^{a}$ Institute of Computational Intelligence \\ Częstochowa University of Technology, ul. Armii Krajowej 36, 42-200 Częstochowa, Poland \\ e-mail:\{lukasz.bartczuk, andrzej.przybyl, krzysztof.cpalka\}@iisi.pcz.pl
}

\begin{abstract}
For many practical weakly nonlinear systems we have their approximated linear model. Its parameters are known or can be determined by one of typical identification procedures. The model obtained using these methods well describes the main features of the system's dynamics. However, usually it has a low accuracy, which can be a result of the omission of many secondary phenomena in its description. In this paper we propose a new approach to the modelling of weakly nonlinear dynamic systems. In this approach we assume that the model of the weakly nonlinear system is composed of two parts: a linear term and a separate nonlinear correction term. The elements of the correction term are described by fuzzy rules which are designed in such a way as to minimize the inaccuracy resulting from the use of an approximate linear model. This gives us very rich possibilities for exploring and interpreting the operation of the modelled system. An important advantage of the proposed approach is a set of new interpretability criteria of the knowledge represented by fuzzy rules. Taking them into account in the process of automatic model selection allows us to reach a compromise between the accuracy of modelling and the readability of fuzzy rules.
\end{abstract}

Keywords: nonlinear modelling, dynamic systems, fuzzy systems, interpretability of fuzzy systems, evolutionary algorithms.

\section{Introduction}

The modelling of real systems and physical phenomena is very important from a theoretical and a practical point of view. It is used to develop control and failure detection systems, communication, analysis of chemical and biological processes, etc. (see, e.g., Boukezzoula et al., 2007; Witkowska and Śmierzchalski, 2012; Xie et al., 2006; Adjrad and Belouchrani, 2007; Huijberts et al., 2000). It aims to ensure that the created model was accurate and computationally undemanding. As a result, it can work in real time (see, e.g., Bagarinao et al., 2003; DeHaan and Guay, 2006; Fei et al., 2011). A desirable feature of the model is also its transparency and interpretability because they guarantee the possibility of a better understanding of the analysed phenomenon (see, e.g., Johansson et al., 2011; Gacto et al., 2011; Rüping, 2006).

It should be noted that real objects are nonlinear in nature and, therefore, to build their models is not a trivial task. It is much easier to build a model

${ }^{*}$ Corresponding author of a linear object. Such models are also much less computationally demanding. The result is that very often nonlinear objects are modelled by means of one or several connected linear models (see, e.g., Murray-Smith and Johansen, 1997; Banerjee et al., 1997). An important advantage of this approach is an easier way to build a model which is based on the theoretical description of the known physical phenomena. The representation of the model is interpretable, thereby these methods are referred to as a white box (see, e.g., Nelles, 2001; Ikonen and Najim, 2001; Roffel and Betlem, 2004). However, it should be noted that, due of the need to adopt simplifying assumptions, these methods are often not adequately accurate.

One way of building models of nonlinear systems is to observe the system response to a given input signal and to attempt to reproduce this dependence in the model (see, e.g., Ljung, 2010; Háber and Keviczky, 1999; Grabowski and Callier, 2001). Such methods are oriented primarily toward achieving high accuracy during reproduction of input-output dependencies, which 
is, however, accomplished at the expense of the lack of interpretability of the obtained model. For this reason, this approach is referred to as a black box. However, in many application areas such an approach is suitable. Examples of methods belonging to that group are neural networks (see, e.g., Tadeusiewicz et al., 2014; Mrugalski, 2014; Tadeusiewicz and Figura, 2011; Salapa et al., 2014; Horzyk and Tadeusiewicz, 2004; Tadeusiewicz, 2010; Puig et al., 2007). They are classified as the so-called computational intelligence methods (see, e.g., Patton et al., 2005; Rutkowski, 2008; Wilamowski, 2005). They are universal approximators, which makes them useful tools for modelling complex, nonlinear dynamic objects (see, e.g., Tan, 2004; Nelles, 2001; Pedro and Dahunsi, 2011). Unfortunately, in neural networks all information about the analysed phenomenon is stored in the form of numerical weights, whose values are determined while forming the model. The result is that obtaining interpretable information about the modelled phenomenon is difficult, if not impossible.

Between the methods belonging to the white box group and those belonging to the black box one there are approaches included in the so-called grey box category (Bohlin, 2006; Kristensen et al., 2004). Their creators try to combine the best features of the previously mentioned methods. The resulting models are based on physical laws describing the analysed phenomena, while their parameters are determined by the analysis of the system's behaviour. Thus, a compromise between accuracy of the model and its interpretability can be reached. Examples of methods belonging to this group are fuzzy systems and neuro-fuzzy systems, also included in the methods of computational intelligence (Gacto et al., 2011; Rutkowski, 2008; Cpałka, 2009b). As opposed to neural networks, in fuzzy systems the information about the internal structure of the model can be easily read because knowledge is represented in a readable form, e.g., as fuzzy rules (Gacto et al., 2011; Rutkowski, 2008; Cpałka, 2009b). The key aspect of the design of a fuzzy system is to determine its parameters, including fuzzy sets present in fuzzy rules. In the literature we can find many approaches that allow us to accomplish this task, among others, gradient methods (Medasani et al., 1998; Rutkowski and Cpałka, 2005), clustering methods (Starczewski et al., 2010; Malchiodi and Pedrycz, 2013), or population based algorithms (Cpałka, 2009a; 2009b; Cpałka et al., 2014; 2013). The latter perform very well in practice because in addition to the shape and the position of the membership function, they also allow us to determine the form of fuzzy rules and a convenient implementation of interpretability criteria.

In this paper we propose a new approach to modelling nonlinear systems, which can be placed between methods from the white and grey boxes. The proposed approach has been applied to weakly nonlinear dynamic systems with linear inputs and nonlinear dynamics (Caughey, 1963). They are important from a practical point of view and are described in Section 2. The main features of the proposed method can be summarized as follows:

- It is based on the linear model and generates deviations from this model. Direct use of the linear model in the areas in which the system characteristics are nonlinear may cause a sharp decline in modelling accuracy. We assume that modelling the deviations from the linear model, i.e., based on linear state equations, significantly reduces or eliminates the effect of the decrease in modelling accuracy. It should be noted that our method is an interesting combination of the classic approach to modelling and the approach utilizing the potential of computational intelligence. Similar solutions have not been discussed in the literature.

- It utilizes neuro-fuzzy systems to generate values of corrections to the existing linear model. In neuro-fuzzy systems knowledge is stored in the form of readable IF-THEN fuzzy rules. In addition, the parameters of these rules can be automatically determined by machine learning. This makes it possible to extract the information in which areas and how the linear model has been improved for greater accuracy. Similar solutions have not been discussed in the literature.

- It uses an evolutionary method for determining the structure and parameters of the neuro-fuzzy systems used. Evolutionary methods are optimization techniques inspired by nature which, owing to their advantages (summarised at the beginning of Section 4), are being dynamically developed. The use of evolutionary methods allowed, among others, parallel optimization of the structure (the form of rules) and parameters of neuro-fuzzy systems, taking into account the adopted interpretability criteria.

- It takes into account new aspects of interpretability of neuro-fuzzy systems during their automatic creation. As mentioned earlier, the use of neuro-fuzzy systems cannot directly guarantee obtaining models which can be easily interpreted. Therefore, in the proposed method we have taken into account constraints in the design of neuro-fuzzy systems to get a model whose knowledge can be easily interpreted.

This paper is organized as follows. Section 2 contains a description of the idea of the proposed method for modelling nonlinear systems. Neuro-fuzzy systems used in modelling nonlinear systems are presented in Section 3. Section 4 describes the method of designing such a system with evolutionary methods. The results 
of simulations are presented in Section 5. The paper is summarized in Section 6.

\section{Idea of the proposed approach}

2.1. Modelling of weakly nonlinear dynamic systems. In the dynamic system the response depends not only on current input values but also on the values of the current state of the system. In a general case the nonlinear system dynamics are described by the following equation:

$$
\frac{\mathrm{d} \mathbf{x}}{\mathrm{d} t}=f(\mathbf{x}, \mathbf{v})
$$

where $\mathbf{x}$ is a vector of state variables, $f(\mathbf{x}, \mathbf{v})$ is a nonlinear function that represents the changes in the system state and $\mathbf{v}$ is the vector of input values. In this paper we focus on the modelling of weakly nonlinear dynamic systems. These are those whose trend of operation is linear. Consequently, their way of operation can be approximated by linear dependencies. For such systems nonlinearities cause a deviation from the linear approximation, which results, e.g., from slight changes in the parameters of certain elements of the circuit, etc. An example of a simple weakly nonlinear dynamic system is an electrical circuit consisting of real (i.e., non-ideal) elements like capacitors, resistors and inductors. In this circuit in the coil with a ferromagnetic core the inductance slightly changes in response to a change in the value of the electric current. Similarly, the resistance, inductance and capacitance change in response to temperature variations. Another example is the kinetic friction coefficient, which can slightly change due to changes in the relative speed of two moving bodies. A practical example is also the asymmetry in the magnetic field distribution in electric motors, which is not included in the widely used analytical models of such systems.

In the literature on the modelling of weakly nonlinear dynamic systems we can often see the following way of their approximation:

$$
\frac{\mathrm{d} \mathbf{x}}{\mathrm{d} t}=f(\mathbf{x}, \mathbf{v}) \approx \mathbf{A} \mathbf{x}+\mathbf{B} \mathbf{v}
$$

where $\mathbf{A}$ is a system matrix (defining the system dynamics, i.e., the impact of the state variable on the state change) and $\mathbf{B}$ is an input matrix (defining the impact of the system input on the state change). Equation (2) can be applied when it is possible to determine the values of matrices $\mathbf{A}$ and $\mathbf{B}$ and the resulting accuracy is sufficient. However, because the obtained accuracy is often not sufficient, new methods of approximation of nonlinear dynamic objects are still being sought. This is realized to simplify the analysis of the model in comparison with, e.g., an analysis of the model that is based on a theoretical description of the known physical phenomena. The simplification is a result of, among other things, the possibility of using well-known methods in the fields of control theory that have been developed for linear systems.

2.2. Modelling of weakly nonlinear systems with linear inputs and nonlinear dynamics. The modelling of weakly nonlinear systems with nonlinear inputs and nonlinear dynamics can be based on the equivalent linearization technique (Caughey, 1963). In this method it is assumed that the general formula describing the model of the system (1) is expressed by the following state equation:

$$
\frac{\mathrm{d} \mathbf{x}}{\mathrm{d} t}=\mathbf{A} \mathbf{x}+\mathbf{B} \mathbf{v}+\eta g(\mathbf{x}, \mathbf{v}),
$$

where $g(\cdot)$ is a function which defines the nonlinearity of the system and $\eta$ determines the impact of function $g(\cdot)$ on the entire object. Equation (3) can be used for modelling any nonlinear system (not only weakly nonlinear systems) because the function $g(\cdot)$ can theoretically represent any nonlinearity. However, determination of the function $g(\cdot)$ for the whole range of operation for the modelled system is difficult, if not possible. For this reason the range of modelling of weakly nonlinear systems is usually limited only to the surroundings of some typical operating point $\left(\mathbf{x}_{s}, \mathbf{v}_{s}\right)$. In some strictly defined range around this point the modelled object behaves in a manner similar to the linear one.

Then the influence of the component $\eta g(\mathbf{x})$ in Eqn. (3) is small, so the equation can be simplified to the form represented by Eqn. (2). Such a class of systems, i.e., when $\eta$ is "small in some sense", may be treated as weakly nonlinear system according to the explanation given by Caughey (1963).

In the equivalent linearization technique, Eqn. 3 can also be represented in alternative form as

$$
\frac{\mathrm{d} \mathbf{x}}{\mathrm{d} t}=\mathbf{A}_{e q} \mathbf{x}+\mathbf{B}_{e q} \mathbf{v}+e(\mathbf{x}, \mathbf{v})
$$

where matrices $\mathbf{A}_{e q}$ and $\mathbf{B}_{e q}$ describe the model of the system considered linear at the operating point $\left(\mathbf{x}_{s}, \mathbf{v}_{s}\right)$ and have the following form:

$$
\left\{\begin{array}{l}
\mathbf{A}_{e q}=\mathbf{A}+\mathbf{P}_{\mathbf{A}} \\
\mathbf{B}_{e q}=\mathbf{B}+\mathbf{P}_{\mathbf{B}} .
\end{array}\right.
$$

In the case of systems with linear inputs and nonlinear dynamics (Schröder, 2000) the matrix $\mathbf{P}_{\mathbf{B}}$ is zero. The correction matrix $\mathbf{P}_{\mathbf{A}}$ is estimated for the operating point considered in such a way that the error term $e(\cdot)$ of the linear approximation is as small as possible. Finally, the model of the weakly nonlinear dynamic system considered in some strictly defined range around some typical operating point $\left(\mathbf{x}_{s}, \mathbf{v}_{s}\right)$ can be written as follows:

$$
\frac{\mathrm{d} \mathbf{x}}{\mathrm{d} t} \approx\left(\mathbf{A}+\mathbf{P}_{\mathbf{A}}\right) \mathbf{x}+\mathbf{B v}
$$


2.3. Modelling of weakly nonlinear dynamic systems with linear inputs and nonlinear dynamics with intelligent correction of the linear model. The values of coefficients of the matrix $\mathbf{P}_{\mathbf{A}}$ depend on the current operating point. The correction matrix values depend on the selected operating point, so they are changing when moving away from this point. This can significantly affect the modelling accuracy. It is the most important drawback of such a modelling method.

Due to the inconvenience described earlier, in this paper it is assumed that the values of the matrix $\mathbf{P}_{\mathbf{A}}$ are not constant but they are functions that take into account the current state $\mathbf{x}$ of the system being modelled, so $\mathbf{A}_{e q}(\mathbf{x})=\mathbf{A}+\mathbf{P}_{\mathbf{A}}(\mathbf{x})$. Due to this, these values may change with the change of the current operating point (belonging to the set of predefined operating points). Taking this fact into account, finally we can write

$$
\frac{\mathrm{d} \mathbf{x}}{\mathrm{d} t} \approx\left(\mathbf{A}+\mathbf{P}_{\mathbf{A}}(\mathbf{x})\right) \mathbf{x}+\mathbf{B v}
$$

In the remainder of this paper we consider only linearisable dynamic models given by (1), which can be described by (7).

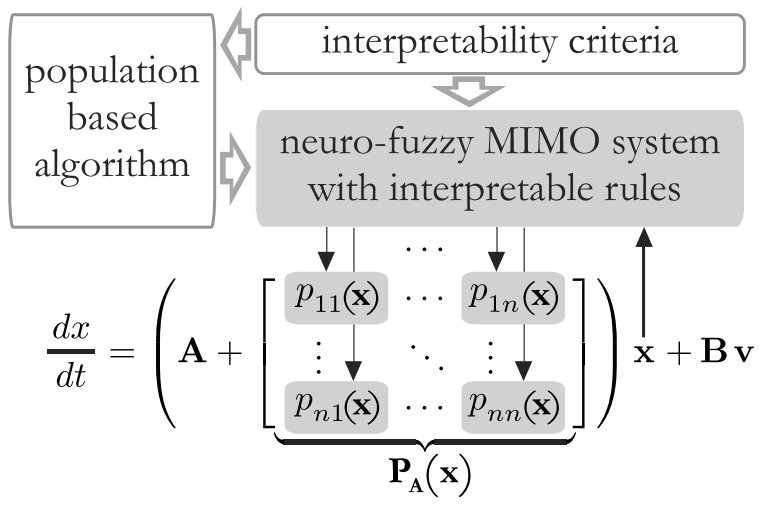

Fig. 1. Idea of the proposed method for correction modelling of weakly nonlinear dynamic systems with linear inputs and nonlinear dynamics.

For generating values of the matrix $\mathbf{P}_{\mathbf{A}}(\mathbf{x})$ in Eqn. (7) we suggest the use of selected methods of artificial intelligence, i.e., fuzzy systems and population based algorithms (see Fig. 1). Other features of the proposed methods can be summarized as follows.

Hallmark 1. They are used to model weakly nonlinear dynamic objects for which the general form of the approximated linear model is known. This means that the values of the matrices $\mathbf{A}$ and $\mathbf{B}$ are known and they result from, e.g., the knowledge of the parameters of the analytical model that approximately describes the system dynamics. This knowledge may result from information about physical properties of materials used for the construction of the modelled system. These properties arise from physical constants (like, e.g., permeability coefficient, heat capacity, etc.) and physical characteristics (like, e.g., the number of turns of inductor, physical size, etc.). The knowledge about parameters of the analytical model may also result from a previously conducted identification procedure using one of the many well-known identification methods (see, e.g., Przybył and Jelonkiewicz, 2003). However, the problem of determining the coefficients of the matrices $\mathbf{A}$ and $\mathbf{B}$ is a separate issue and is not within the scope of this paper. When the proposed method has the general form of an approximated linear model, it is able to automatically select the values of the correction matrix $\mathbf{P}_{\mathbf{A}}$ to improve the accuracy of the modelling, taking into account individual characteristics of the modelled real-world object. The correction matrix $\mathbf{P}_{\mathbf{A}}$ can change with the change of the current operating point.

Hallmark 2. They concern the modelling of weakly nonlinear dynamic systems, for which the matrices $\mathbf{A}$ and $\mathbf{B}$ are known and the model which uses those matrices is correct in terms of theoretical and practical assumptions. This makes it possible to focus on practical aspects of the operation and omit the need for a theoretical analysis of some special situations which result from ambiguity or discontinuities of the system being modelled. Such an analysis would be necessary in the modelling of dynamic systems in the general case. For this reason the ideas presented in this paper are limited to weakly nonlinear systems with linear inputs and nonlinear dynamics.

Hallmark 3. They use the possibilities of fuzzy sets and system theory. In particular, a fuzzy system with multiple inputs and multiple outputs. The current values of the state vector are used as the input values of the system, and on this basis the system generates values of the correction matrix $\mathbf{P}_{\mathbf{A}}$. The number of the system outputs depends on the dimensions of the correction matrix $\mathbf{P}_{\mathbf{A}}$. This approach has a very important advantage-a readable form of the fuzzy IF...THEN... rules allows describing the source of nonlinearity (a deviation from the approximated linear model) occurring in the modelled system. It should be noted that in this method any known architecture of the fuzzy system can be used (in particular a typical Mamdani type architecture described in Section 3). The type of fuzzy system applied is not a novel element of this paper.

Hallmark 4. They use automatic selection of values of the matrix $\mathbf{P}_{\mathbf{A}}$ realized using the capabilities of supervised learning (see, e.g., Rutkowski, 2008). This is done in a manner typical for computational intelligence systems, such as artificial neural networks or neuro-fuzzy systems. We assume that in order to train the system the data from non-invasive identification of a modelled real world 
system are used. The method employed to train the neuro-fuzzy system is described in detail in Section 4. It should be noted that the method is known in the literature and is not a novel element of this paper (an appropriate description of mathematical analysis and rigorous design methods for fuzzy control systems may be found in the works of Kluska (2009; 2015)). However, a novel element of this paper is the fact that the fuzzy rules describing sources of nonlinearity are formed in a very flexible way and the algorithm promotes readable rules. This makes it possible, e.g., to detect that the first element of the matrix $\mathbf{P}_{\mathbf{A}}$ is affected only by the last element of the state vector $\mathbf{x}$

Hallmark 5. They take into account appropriately formulated criteria for the clarity of fuzzy rules used to model the correction matrix $\mathbf{P}_{\mathbf{A}}$ (described in Section 3.1). It is worth noting that in many papers on nonlinear modelling fuzzy systems are used directly for modelling dependence $f(\mathbf{x}, \mathbf{v})$ in Eqn. (1). In some applications this approach works well, but if the problem is complex and then, in order to achieve reasonable accuracy, multiple rules are needed. A large number of rules makes them very difficult to analyse. In the proposed approach deviations from the approximated linear model can be described more easily by fuzzy rules than whole nonlinear object. Moreover, in this paper some new readability criteria of fuzzy rules are formulated and used in the training process in order to increase the readability of rule-based notation of the correctional matrix $\mathbf{P}_{\mathbf{A}}$.

\section{Neuro-fuzzy systems for modelling nonlinear systems}

In this section, a multiple-input multiple-output (MIMO) fuzzy system is described. The parameters of this system are chosen as a result of a population based (supervised) algorithm which is presented in Section 4. These parameters can also be set by a gradient algorithm (analogously as, for example, weights in artificial neural networks). For this reason, in the sequel the fuzzy system considered will be called a neuro-fuzzy system. Such a system is based on IF-THEN fuzzy rules, in which the values of inputs and outputs linguistic variables are characterized by fuzzy sets (see, e.g., Rutkowski and Cpałka, 2005; Rutkowski, 2008).

3.1. Multiple input multiple output neuro-fuzzy system. The utilized MIMO neuro-fuzzy system preforms a mapping $\mathbf{W} \rightarrow \mathbf{Z}$, where $\mathbf{W} \subset \mathbb{R}^{n}, \mathbf{Z} \subset \mathbb{R}^{m}$ (Rutkowski, 2008). Such a system is composed of several cooperating functional blocks. The fuzzifier realizes a mapping from a crisp input space $\mathbf{W}$ to the fuzzy sets defined in $\mathbf{W}$. The most commonly used fuzzifier is the singleton one (see, e.g., Rutkowski, 2008), which maps input values $\overline{\mathbf{W}}=\left[\bar{w}_{1}, \ldots, \bar{w}_{n}\right] \in \mathbf{W}$ into a fuzzy set $A^{\prime} \subseteq \mathbf{W}$ characterized by a membership function

$$
\mu_{A^{\prime}}(\mathbf{w})= \begin{cases}1 & \text { if } \quad \mathbf{w}=\overline{\mathbf{w}} \\ 0 & \text { if } \quad \mathbf{w} \neq \overline{\mathbf{w}}\end{cases}
$$

A collection $\mathcal{A}_{i}=\left\{A_{i, 1}, \ldots, A_{i,\left|\mathcal{A}_{i}\right|}\right\}$ of fuzzy sets is defined on $\mathbf{W}_{i}$ for each system input $i=1, \ldots, n$, where $\left|\mathcal{A}_{i}\right|$ is the number of elements of collection $\mathcal{A}_{i}$ and $n$ is the number of system inputs. In turn, a collection $\mathcal{B}_{j}=\left\{B_{j, 1}, \ldots, B_{j,\left|\mathcal{B}_{j}\right|}\right\}$ of fuzzy sets is defined on $\mathbf{Z}_{j}$ for each system input $j=1, \ldots, m$, where $\left|\mathcal{B}_{j}\right|$ is the number of elements of collection $\mathcal{B}_{j}$ and $m$ is the number of system outputs. Each fuzzy set $A_{i, l}$ is characterized by membership function $\mu_{A_{i, l}}\left(w_{i}\right), l=1, \ldots,\left|\mathcal{A}_{i}\right|$, and each fuzzy set $B_{j, l}$ is characterized by membership function $\mu_{B_{j, l}}\left(z_{j}\right), l=1, \ldots,\left|\mathcal{B}_{j}\right|$. Thus the fuzzy rule base can be defined as a collection $\mathcal{R}=R^{1}, \ldots, R^{|\mathcal{R}|}$, where $|\mathcal{R}|$ is the number of elements of this collection. Each rule can be written in the following form:

$$
R^{(k)}:\left\{\begin{array}{l}
\text { IF } w_{1} \text { IS } A_{1}^{k} \text { AND } \ldots \text { AND } w_{n} \text { IS } A_{n}^{k} \\
\text { THEN } z_{1} \text { IS } B_{1}^{k} \text { AND } \ldots \text { AND } z_{m} \text { IS } B_{m}^{k}
\end{array}\right.
$$

where $\mathbb{W}=\left[w_{1}, \ldots, w_{n}\right] \in \mathbf{W}, \mathbb{Z}=\left[z_{1}, \ldots, z_{m}\right] \in \mathbf{Z}$, $A_{i}^{k} \in \mathcal{A}_{i}$ is a fuzzy set from collection $\mathcal{A}_{i}$ used in the $k$-th rule and $B_{j}^{k} \in \mathcal{B}_{j}$ is a fuzzy set from collection $\mathcal{B}_{j}$ used in the $k$-th rule.

Fuzzy inference determines a mapping from the fuzzy set in input space $\mathbf{W}$ to the fuzzy sets in output space Z . Each of the rules (9) generates fuzzy sets $\bar{B}_{j}^{k} \subset$ $\mathbf{Z}$ given by the compositional rule of inference:

$$
\bar{B}_{j}^{k}=A^{\prime} \circ\left(\mathbf{A}^{k} \rightarrow B_{j}^{k}\right),
$$

where $\mathbf{A}^{k}=A_{1}^{k} \times \cdots \times A_{n}^{k}$, and $\mathbf{A}^{k} \rightarrow B_{j}^{k}$ means fuzzy implication (Rutkowski, 2008; Rutkowski and Cpałka, 2005). The membership function characterizing set $\bar{B}_{j}^{k}$ can be defined by sup-star composition (denoted by "o") and expressed as

$$
\begin{aligned}
& \mu_{\bar{B}_{j}^{k}}\left(z_{j}\right) \\
& \quad=\sup _{\mathbf{w} \in \mathbf{W}}\left(\mathcal{T}\left\{\mu_{A^{\prime}}(\mathbf{w}), \mu_{\mathbf{A}^{k} \rightarrow B_{j}^{k}}\left(\mathbf{w}, z_{j}\right)\right\}\right),
\end{aligned}
$$

where $t$-norm $\mathcal{T}\{\cdot\}$ is a generalization of the usual two-valued logical conjunction (see, e.g., Rutkowski, 2008). It should be noted that for a singleton fuzzifier (8) the formula (11) becomes

$$
\begin{aligned}
\mu_{\bar{B}_{j}^{k}}\left(z_{j}\right) & =\mu_{\mathbf{A}^{k} \rightarrow B_{j}^{k}}\left(\mathbf{w}, z_{j}\right) \\
& =\mathcal{I}_{j}\left(\mu_{\mathbf{A}^{k}}(\overline{\mathbf{w}}), \mu_{B_{j}^{k}}\left(z_{j}\right)\right),
\end{aligned}
$$


where $\mathcal{I}_{j}(\cdot)$ is an inference operator associated with the $j$-th system output. It can be defined as a $t$-norm (Mamdani type systems) or as a logical implication (logical type systems). In this paper we consider Mamdani type systems (see, e.g., Rutkowski, 2008), so we use the $t$-norm as an inference operator (e.g., the algebraic minimum). It should be noted that in our method we assume that we can use a different inference operator for each system output. This is realized in order to increase the flexibility of modelling.

The last functional block of the neuro-fuzzy system considered, i.e., the defuzzifier, performs a mapping from the collection of fuzzy sets $\bar{B}_{j}^{k}$ to crisp points $\overline{z_{j}}$ in $\mathbf{Z} \subset$ $\mathbb{R}^{m}$. This is accomplished by determining the point $z_{j}^{k}$ for each fuzzy set $B_{j}^{k}$, where its membership function takes the value of 1 , that is, $\mu_{B_{j}^{k}}\left(z_{j}^{k}\right)=1$, and also by using an appropriate method of defuzzification, e.g., the centre of average:

$$
\overline{z_{j}}=\frac{\sum_{k=1}^{|\mathcal{R}|} z_{j}^{k} \cdot \mu_{B_{j}^{k}}\left(z_{j}^{k}\right)}{\sum_{k=1}^{|\mathcal{R}|} \mu_{B_{j}^{k}}\left(z_{j}^{k}\right)} .
$$

It should be noted that in a neuro-fuzzy system of the form (13) any membership function with a single core value can be applied. In the simulations (see Section 5) we use Gaussian membership functions (see, e.g., Rutkowski, 2008) for input fuzzy sets and singleton membership functions of the form (8) for output fuzzy sets. Gaussian functions describe well the phenomena occurring in nature and in real industrial processes. Singleton membership functions simplify the structure of the system used because the values $\bar{z}_{j}$ are independent of the type of the membership function of output fuzzy sets. Their use also makes the Mamdani type fuzzy system equivalent to a zero-order Takagi-Sugeno type fuzzy system (see, e.g., Jang and Sun, 1995). If the use of a multivalue core membership function (e.g., trapezoidal) is necessary, the defuzzification method should be changed.

\subsection{Interpretability of neuro-fuzzy systems.} Neuro-fuzzy systems are very often used to model various physical phenomena (Babuska and Verbruggen, 2003; Czekalski, 2006; Łęski, 2003; Li and Chiang, 2012; Quah and Quek, 2006). As shown, e.g., by Gacto et al. (2011), the resulting models can be classified into one of two groups:

1. Precise fuzzy models developed in order to maximize the accuracy of the representation of the modelled phenomenon. Models of this group are often characterized by a large number of fuzzy rules and limited possibilities to assign linguistic labels to fuzzy sets (this is difficult or even impossible).
2. Interpretable (linguistic) fuzzy models that reflect the behaviour of a real system in a manner as simple as possible to understand.

It should be noted that these goals are contradictory and fulfilling both of them is not fully possible (Gacto et al., 2011). Therefore, during the last few years many researchers focused on obtaining a compromise between accuracy and interpretability of fuzzy systems (see, e.g., Zhou and Gan, 2008; Casillas et al., 2003; Di Nuovo and Ascia, 2013; Ishibashi and Lucio Nascimento, Jr., 2013; Shukla and Tripathi, 2013; Juang and Chen, 2013; Lughofer, 2013; Johansen et al., 2000).

In the literature, interpretability is considered to be a complexity of fuzzy models and their semantics both on fuzzy rule and fuzzy partition levels. Interpretability of fuzzy models can be provided in many ways, but restrictions on the learning process are imposed most commonly (see, e.g., Lughofer, 2013; Cpałka et al., 2014; Shukla and Tripathi, 2013; Ishibashi and Lucio Nascimento, Jr., 2013).

The interpretability assumptions derived from the literature and the proposed criteria resulting from them and used in this paper are shown below.

Postulate 1. The number of inputs, rules as well as their antecedents and consequents should be as small as possible.

While designing a fuzzy system it may occur that some of the available inputs, fuzzy sets and rules are redundant, i.e., dropping them does not negatively affect the accuracy of the resulting model. In such a case, when rejecting these elements, we get a system with a lower complexity, and therefore with a rule base easier to interpret. Thus the first proposed interpretability criterion is defined as the ratio of the number of elements of the fuzzy system identified automatically by an algorithm and the greatest possible number of its elements. The greatest number of the system's elements results from including all the available inputs and the allowable number of rules. The criterion considered can be written as follows:

$$
I_{1}=\frac{n+\sum_{i=1}^{|\mathcal{R}|}\left|\mathcal{A}_{i}\right|+|\mathcal{R}|}{\tilde{n}+\tilde{n} \cdot|\tilde{\mathcal{A}}|+|\tilde{\mathcal{R}}|},
$$

where $\tilde{n}$ is the number of all available system inputs, $|\tilde{\mathcal{A}}|$ is the predetermined largest number of fuzzy sets specified for each system's input (assuming that this number is the same for each input), $|\tilde{\mathcal{R}}|$ is the predetermined largest number of rules from which the fuzzy system can be composed, $n$ is the number of inputs used in the neuro-fuzzy system (where $n \leq \tilde{n}$ ), $\left|\mathcal{A}_{i}\right|$ is the number of fuzzy sets specified for the $i$-th input of the system (where $\left.\left|\mathcal{A}_{i}\right| \leq|\tilde{\mathcal{A}}|, i=1, \ldots, n\right),|\mathcal{R}|$ is the number of rules used in the system (where $|\mathcal{R}| \leq|\tilde{\mathcal{R}}|$ ). 
a) $\quad \mu\left(w_{1}\right)$

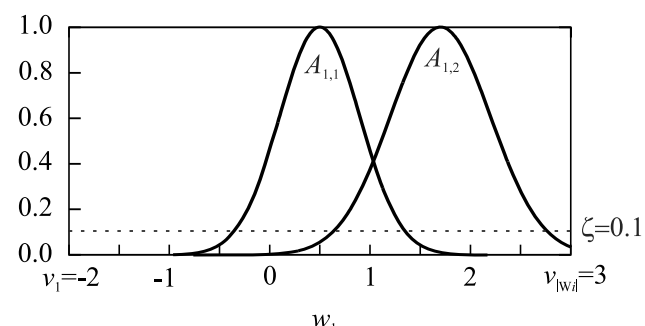

c) $\quad \mu\left(w_{1}\right)$

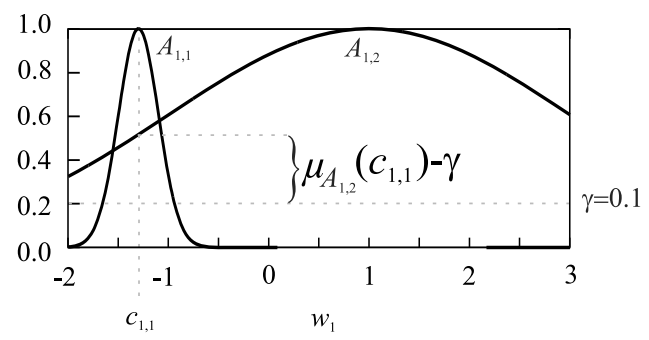

b) $\quad \mu\left(w_{1}\right)$

$g_{1,1} \quad \mu_{A_{1,1}}\left(g_{1,1}\right)-\bar{\kappa}$

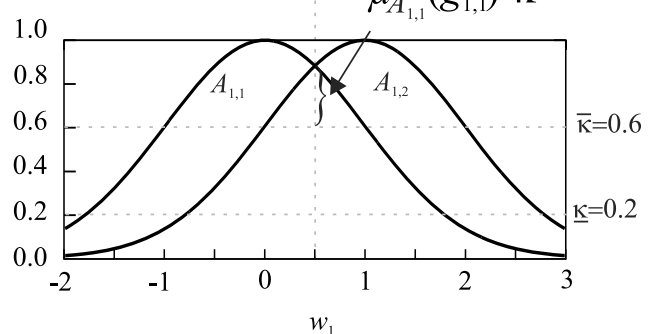

d) $\mu\left(w_{1}\right)$

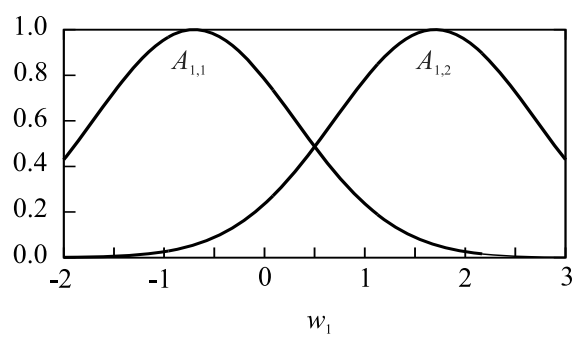

Fig. 2. Examples of fuzzy partitions: fuzzy sets do not cover all the universe of the discourse-unfulfilled Postulate 2(a), fuzzy sets are overlapping too much-unfulfilled Postulate 3(b), fuzzy set $A_{1,1}$ is contained in set $A_{1,2}$ in a too high degree-unfulfilled Postulate 4(c), fuzzy partition that fulfills all the postulates (d).

Postulate 2. In the obtained fuzzy model, fuzzy sets should cover the whole universe of discourse.

The purpose of the criterion is making the whole universe of discourse $\mathbf{W}_{i}$ of each input covered by fuzzy sets, and the membership of any point $w \in \mathbf{W}$ of this universe, in at least one fuzzy set, not lower than $\zeta \in[0,1]$. An example of a fuzzy partition not meeting this criterion is presented in Fig. 2(a). Assuming that the universe of discourse $\mathbf{W}_{i}$ for each input is uniformly divided to $\left|\mathbf{W}_{i}\right|$ points $v_{i, z} \in\left|\mathbf{W}_{i}\right|, z=1, \ldots,\left|\mathbf{W}_{i}\right|$, the criterion considered can be defined as an average number of points in which the degree of membership in each fuzzy set generated for the $i$-th input is not greater than $\zeta$. This can be expressed by the formula.

$$
I_{2}=\frac{1}{n} \sum_{i=1}^{n} \frac{\sum_{z=1}^{\left|\mathbf{W}_{i}\right|}\left\{\begin{array}{cc}
1 & \text { if } \max _{l=1, \ldots,|\tilde{\mathcal{A}}|}\left(\mu_{A_{i, l}}\left(v_{i, z}\right)\right)<\zeta \\
0 & \text { otherwise }
\end{array}\right.}{\left|\mathbf{W}_{i}\right|} .
$$

Postulate 3. In the obtained fuzzy model, fuzzy sets should not significantly overlap.

The purpose of this criterion is to reduce the overlapping of neighbouring fuzzy sets, thus ensuring their distinguishability (the possibility to give them appropriate semantic meaning). An example of a fuzzy partition not meeting this criterion is presented in Fig. 2(b). The considered criterion can be defined as an average deviation of the degree of membership specified at the intersection point of subsequent membership functions from interval $[\underline{\kappa}, \bar{\kappa}](\underline{\kappa} \in[0,1], \bar{\kappa} \in[0,1], \underline{\kappa}<\bar{\kappa})$, and presented as follows:

$$
\sum_{l=1}^{\left|\mathcal{A}_{i}\right|-1}\left\{\begin{array}{c}
\mu_{A_{i, l}}\left(g_{i, l}\right)-\bar{\kappa} \\
\text { if } \mu_{A_{i, l}}\left(g_{i, l}\right)>\bar{\kappa} \\
\underline{\kappa}-\mu_{A_{i, l}}\left(g_{i, l}\right) \\
\text { if } \mu_{A_{i, l}}\left(g_{i, l}\right)<\underline{\kappa} \\
0 \quad \text { otherwise }
\end{array}\right.
$$

where $[\underline{\kappa}, \bar{\kappa}] \subseteq[0,1]$ is a predetermined interval to which the degree of membership specified at the intersection point of subsequent membership functions should belong, $g_{i, l} \in \mathbf{W}_{i}$ is the point from the domain of the $i$-th system input, where the adjacent membership functions $\mu_{A_{i, l}}\left(w_{i}\right)$ and $\mu_{A_{i, l+1}}\left(w_{i}\right)$ intersect (i.e., achieve the same value: $\left.\mu_{A_{i, l}}\left(g_{i, l}\right)=\mu_{A_{i, l+1}}\left(g_{i, l}\right)\right)$.

Postulate 4. In the obtained model, the value of any membership function in the core of other membership functions should be low.

This criterion is intended to ensure that the system which has achieved full membership in to fuzzy set belongs at most in degree $\gamma \in[0,1]$ to other fuzzy sets generated for the $i$-th input. An example of a fuzzy partition not meeting this criterion is presented in Fig. 2(c). The considered criterion can be defined as an average 
difference between the threshold value $\gamma$ and the value of membership function $\mu_{A_{i, l}}$ determined at points $x_{i, l^{\prime}}$, $\left(l^{\prime}=1, \ldots,\left|\mathcal{A}_{i}\right|, l^{\prime} \neq l\right)$, where the other membership functions reach the value of 1 . This can be described by the following formula:

$$
I_{4}=\frac{1}{n} \sum_{i=1}^{n} \frac{\sum_{l=1}^{\left|A_{i}\right|\left|A_{i}\right|} \sum_{\substack{l^{\prime}=1 \\
l^{\prime} \neq l}}\left\{\begin{array}{l}
\mu_{A_{i, l}}\left(c_{i, l^{\prime}}\right)-\gamma \\
\text { if } \mu_{A_{i, l}}\left(x_{i, l^{\prime}}\right)>\gamma \\
\text { otherwise }
\end{array}\right.}{\left|A_{i}\right|},
$$

where $c_{i, l^{\prime}} \in \mathbf{W}_{i}$ is the point where membership function $\mu_{A_{i, l^{\prime}}}(w)$ reaches the value of 1 , i.e., $\mu_{A_{i, l^{\prime}}}\left(c_{i, l^{\prime}}\right)=1$, $\gamma$ is a predefined maximum value which the membership function can reach at the core of other membership functions generated for the $i$-th input.

It should be noted that proposed interpretability postulates were adapted to the specifics of neuro-fuzzy systems of the form (13) and described in Section 3.1. They can also be easily adapted to a specific membership function. However, we abandon the presentation of specific equations for different types of membership functions because their extensive notation impedes their readability.

All the presented criteria were designed in such a way that they can be used as an evaluation function of solutions in the process of designing the neuro-fuzzy system. Therefore, all of them take values from the interval $[0,1]$. At the same time the aim is to achieve a solution for which the criteria would be as small as possible. The interpretability of the rule base of such a solution would then be as large as possible.

The usage of the described criteria in order to enhance the interpretability of a fuzzy system (presented in Section 2) will be shown in the next section.

\section{Design of neuro-fuzzy systems for nonlinear systems modelling using an evolutionary strategy}

In literature we can find many methods to design a structure and select parameters of neuro-fuzzy systems (see, e.g., Kim et al., 2006; Wang et al., 2005; Angelov and Filev, 2004; Medasani et al., 1998; Rutkowski and Cpałka, 2005; Starczewski et al., 2010; Malchiodi and Pedrycz, 2013; Cpałka, 2009a; 2009b; Cpałka et al., 2014; 2013). In this paper we used the $(\lambda+\mu)$ evolutionary strategy, which belongs to the group of population based algorithms. All population based algorithms are methods for solving problems (mostly optimization ones) inspired by natural evolution. Population based algorithms differ from traditional optimization methods, among other things, in that (a) they do not directly process the task parameters but their encoded form, (b) the searching of the solution space does not start at one point but from their population, (c) they use only an objective function rather than its derivatives, (d) they use probabilistic rather than deterministic selection rules. Consequently, they have an advantage over other optimization techniques like, e.g., analytical, inspection and random methods (see, e.g., Forst and Hoffmann, 2010; Kroese et al., 2011).

Aspects of construction of neuro-fuzzy systems with the use of population based algorithms are known in the literature. Those algorithms were used, among other things, for the following:

1. The tuning of knowledge bases, i.e., to adjust the shape and parameters of membership functions of inputs and output fuzzy sets. In this case it is assumed that the rule base is predefined and unchanged during the tuning process (Setnes and Roubos, 2000; Gabryel and Rutkowski, 2006; Cpałka, 2009a).

2. Rule base selection, i.e., to adjust the number and the form of fuzzy rules (employed inputs and fuzzy sets occurring in the antecedents and consequents of the rules). In this case it is assumed that the shape and parameters of fuzzy sets are predefined and unchanged during the selection process (Ishibuchi and Yamamoto, 2004; Cordón et al., 2001; Cpałka et al., 2014).

3. Simultaneous tuning of the knowledge base and rule base selection (Homaifar and McCormick, 1995; Wu and Liu, 2000; Shill et al., 2011; Cordón, 2011).

In our proposed approach we assume that an evolutionary strategy is used to select the components of the rule base and to tune the parameters of membership functions. It is worth noting that the selection of the structure and parameters of neuro-fuzzy systems can be also performed by another population algorithm (e.g., a genetic algorithm). The training of the system can be also performed by any gradient algorithm, e.g., the back propagation algorithm (see, e.g., Rutkowski and Cpałka, 2005). However in this case only system parameters can be set with the constant structure indicated by the designer. So it is not a convenient solution.

The first step of the $(\lambda+\mu)$ evolutionary strategy is to generate the initial population Pop that contains $\mu$ individuals (Section 4.2). Next, the temporary population Temp with $\lambda$ individuals (where $\lambda>$ $\mu$ ) is randomly created by using a reproduction operator. Genetic operators like mutation are used with individuals belonging to that temporary population (ensuring exploitation and exploration of the search space). As a result, a population Off is obtained with the same number of individuals as the Temp population. A new parental population Pop is created by a choice of $\mu$ 
best individuals from the combined populations Pop and Temp. Thus, the individuals from the new population Pop are not worse than those from the base population (in terms of the evaluation function). More information about the evolutionary strategy can be found in the literature (see, e.g., Rutkowski, 2008; Eiben and Smith, 2008).

4.1. Chromosome structure. In order to encode the information about the neuro-fuzzy system (13) in a chromosome, we use the Pittsburgh approach (Wang et al., 2005; Rutkowski, 2008; Cpałka, 2009b; Cordón et al., 2001), in which a single chromosome contains information about the entire system. In the structure of a single chromosome $\mathbf{C}_{c h}$ the following four groups of genes can be isolated:

$$
\mathbf{C}_{c h}=\left\{\begin{array}{ll}
\mathbf{C}_{c h}^{\text {params }} & : \text { fuzzy system parameters } \\
\mathbf{C}_{c h}^{\text {sets }} & : \text { fuzzy sets parameters } \\
\mathbf{C}_{c h}^{\text {rules }} & : \text { structure of fuzzy rules } \\
\mathbf{C}_{c h}^{\text {usage }} & : \text { usage of rules and inputs }
\end{array}\right\},
$$

where $c h=1, \ldots, \mu$ stands for the parental population and $c h=1, \ldots, \lambda$ for the temporal one.

Information about each of the specified groups of genes present in the chromosome (18) can be summarized as follows:

1. Genes encoding the type of operators $\mathbf{C}_{c h}^{\text {params }}$ store integer values determining the kind of inference operator used for each output of the neuro-fuzzy system (13):

$$
\mathbf{C}_{c h}^{\text {params }}=\left(p_{1}, \ldots, p_{\tilde{m}}\right),
$$

where $p_{j}(j=1, \ldots, \tilde{m})$ takes values from $\{1,2,3\}$ (1 means a minimum type inference operator, 2 means an algebraic type inference operator and 3 means a Łukasiewicz type inference operator), and $\tilde{m}$ is the number of the available inputs of the fuzzy system. Of course, the set of the operators considered can be flexibly modified.

2. Part $\mathbf{C}_{c h}^{\text {sets }}$ of the chromosome encodes information about the parameters of fuzzy sets from collections $\mathcal{A}_{i}$ and $\mathcal{B}_{j}$ defined on domains $\mathbf{W}_{i}$ and $\mathbf{Z}_{j}$, respectively. Its length depends on the chosen shape of membership functions and the predefined maximum number of elements of collections $\mathcal{A}_{i}$ and $\mathcal{B}_{j}$. When the Gaussian membership function is used for inputs and the singleton membership function is used for outputs of the system, part $\mathbf{C}_{c h}^{\text {sets }}$ of the chromosome can be described by the following formula:

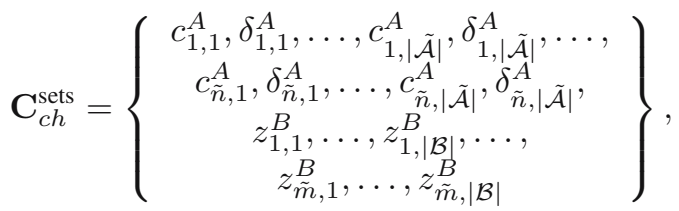

where $c_{i, l}^{A}, \delta_{i, l}^{A}$ are the centres and widths of the Gaussian membership function $(i=1, \ldots, \tilde{n}$, $l=1, \ldots,|\tilde{\mathcal{A}}|)$, respectively, and $z_{j, l}^{B}$ describes the position of the singleton membership function $(j=$ $1, \ldots,|\mathcal{B}|)$ representing the output fuzzy set $B_{j, l}$.

3. Part $\mathbf{C}_{c h}^{\text {rules }}$ of the chromosome encodes information about the fuzzy rule base. We assume that each rule $R^{k}, k=1, \ldots,|\mathcal{R}|$, is composed of a maximum available number of inputs $\tilde{n}$ and outputs $\tilde{m}$, which requires $\tilde{n}+\tilde{m}$ genes. Each of the genes determines the number of the fuzzy set occurring in the antecedents and consequents of the rule $(l=$ $1, \ldots,|\tilde{\mathcal{A}}|$ or $l=1, \ldots,|\mathcal{B}|)$ :

$$
\mathbf{C}_{c h}^{\text {rules }}=\left\{\begin{array}{c}
r i_{1}^{1}, \ldots, r i_{\tilde{n}}^{1}, r o_{1}^{1}, \ldots, r o_{\tilde{m}}^{1}, \\
\ldots \\
r i_{1}^{\tilde{N}}, \ldots, r i_{\tilde{n}}^{\tilde{N}}, r o_{1}^{\tilde{N}}, \ldots, r o_{\tilde{m}}^{\tilde{N}}
\end{array}\right\}
$$

where $r i_{i}^{k} \in\{-1,0,|\tilde{\mathcal{A}}|\}$ is the number of the fuzzy set used in the $k$-th rule for the $i$-th input of the system (the value -1 means that the premise does not occur in the rule), $r o_{j}^{k} \in\{0,|\mathcal{B}|\}$ is the number of the fuzzy set used in the $k$-th rule for the $j$-th output of the system $(j=1, \ldots, \tilde{m}),|\tilde{\mathcal{R}}|$ is the predefined maximum number of rules. In this paper we assume that only the premises can be disabled, because disabling conclusions (in nonlinear modelling) contributes, among other things, to a significant reduction of rules readability.

4. The last part $\mathbf{C}_{c h}^{\text {usage }}$ of the chromosome is a binary vector indicating which rules (out of $|\tilde{\mathcal{R}}|$ ) are considered in the system:

$$
\mathbf{C}_{c h}^{\text {usage }}=\left\{i s_{1}, \ldots, i s_{\tilde{n}}, r s_{1}, \ldots, r s_{|\tilde{\mathcal{R}}|}\right\},
$$

where $i s_{i} \in\{0,1\}$ determines the use of a particular input (when a gene takes on the value of 1 , the corresponding input of the fuzzy system becomes active), while $r s_{k} \in\{0,1\}$ determines the use of a particular rule, $i=1, \ldots, \tilde{n}, k=1, \ldots,|\tilde{\mathcal{R}}|$ (when a gene takes on the value of 1 , the corresponding rule is taken into account during the operation of the fuzzy system). 
4.2. Chromosome initialization. As already mentioned, the purpose of the initialization step is to set the values of genes in the first population of the evolutionary strategy. In the proposed method (and in simulations) we work on the following assumptions about this operation:

1. All inputs and rules are active, that is, $\mathbf{C}_{c h}^{\mathrm{usage}}=$ $(1,1, \ldots, 1)$.

2. All rules are full, that is, there is no -1 value in part $\mathbf{C}_{c h}^{\text {rules }}$ of the chromosome. This can be denoted with auxiliary notation: $\mathbf{C}_{c h}^{\text {rules }}\left\{r i_{i}^{k}\right\} \neq-1$ and $\mathbf{C}_{c h}^{\text {rules }}\left\{r o_{j}^{k}\right\} \neq-1(k=1, \ldots,|\tilde{\mathcal{R}}|, i=$ $1, \ldots, \tilde{n}, j=1, \ldots, \tilde{m})$, which will be used hereafter. This notation allows reference to the part of the chromosome given in curly brackets.

3. For each input and output, rules contain a random combination of input fuzzy sets from collection $\tilde{\mathcal{A}}$ and output fuzzy sets from collection $\mathcal{B}$ (generated according to the uniform distribution).

4. For each input and output, fuzzy sets are uniformly distributed on the universe of the discourse. Therefore, the centres of input fuzzy sets can be determined from

$$
\mathbf{C}_{c h}^{\text {sets }}\left\{c_{i, l}^{A}\right\}=\underline{\mathbf{W}_{i}}+l \frac{\left(\overline{\mathbf{W}_{i}}-\underline{\mathbf{W}_{i}}\right)}{|\tilde{\mathcal{A}}|},
$$

and their widths can be computed with the following formula:

$$
\mathbf{C}_{c h}^{\text {sets }}\left\{\sigma_{i, l}^{A}\right\}=\frac{\overline{\mathbf{W}_{i}}-\underline{\mathbf{W}_{i}}}{2(|\tilde{\mathcal{A}}|-1)},
$$

where $\mathbf{W}_{i}, \overline{\mathbf{W}_{i}}$ are respectively the lower and upper limits for the $i$-th input of the system. The placement of output fuzzy sets can be determined analogously:

$$
\mathbf{C}_{c h}^{\text {sets }}\left\{z_{j, l}^{B}\right\}=\underline{\mathbf{Z}_{i}}+\frac{l\left(\overline{\mathbf{Z}_{i}}-\underline{\mathbf{Z}}_{i}\right.}{|\tilde{\mathcal{B}}|},
$$

where $\mathbf{Z}_{i}, \overline{\mathbf{Z}_{i}}$ are respectively the lower and upper limits for the $j$-th output of the system.

4.3. Evolution of parameters of fuzzy sets. The purpose of the evolutionary strategy used to tune the parameters of membership functions is to make such a selection of their values as to get a system with the greatest possible accuracy while maintaining the interpretability criteria described in Section 3.2. In this process, self-adaptation of the mutation range operator has been used (Fogel, 2006; Eiben and Smith, 2008; Cpałka, 2009 b). For this purpose, for each gene of part $\mathbf{C}_{c h}^{\text {sets }}$ of the chromosome a mutation range value is introduced. This value can be described by the following formula:

$$
\sigma_{c h}^{\mathrm{sets}}=\left\{\sigma_{c h, 1}^{\mathrm{sets}}, \ldots, \sigma_{c h, L}^{\mathrm{sets}}\right\},
$$

where $L=(\tilde{m}+\tilde{n}) \cdot|\tilde{\mathcal{A}}|$ is the number of genes in part $\mathbf{C}_{c h}^{\text {sets }}$ of the chromosome, $c h=1, \ldots, \lambda$ for the temporary population. Taking into account the mutation range $\sigma_{c h}^{\text {sets }}$, the mutation operation can be written as follows:

$$
\sigma_{c h, g}^{\text {sets }^{\prime}}=\sigma_{c h, g}^{\mathrm{sets}} \exp \left(\tau^{\prime} N(0,1)+\tau N_{c h, g}(0,1)\right)
$$

and

$$
\mathbf{C}_{c h, g}^{\text {sets }^{\prime}}=\mathbf{C}_{c h, g}^{\text {sets }}+\sigma_{c h, g}^{\text {sets }^{\prime}} N_{c h, g}(0,1),
$$

where $\sigma_{c h, g}^{\text {sets }}, \sigma_{c h, g}^{\text {sets' }}$ are the current and the new value of the mutation range for the $c h$-th chromosome and the $g$-th gene, $(g=1, \ldots, L), N(0,1)$ is a random number from the standard normal distribution, and $N_{c h, g}(0,1)$ is a random number from the standard normal distribution generated for the $c h$-th chromosome and $g$-th gene, $\tau^{\prime}=$ $1 / \sqrt{2 L}$ and $\tau=1 / \sqrt{2 \sqrt{L}}$ mean predefined constants chosen before the evolutionary process (Eiben and Smith, 2008). Since the mutation operator modifies the values of all genes from part $\mathbf{C}_{c h}^{\text {sets }}$ of the chromosome in each iteration of the algorithm, we drop the use of the crossover operator. The validity of such an approach is confirmed by simulations and suggestions of other authors (Fogel and Atmar, 1990).

4.4. Evolution of the structure of the fuzzy system. The structure of the fuzzy system is encoded in parts $\mathbf{C}_{c h}^{\text {rules }}$ and $\mathbf{C}_{c h}^{\text {usage }}$ of chromosome $\mathbf{C}_{c h}$. Because genes in $\mathbf{C}_{c h}^{\text {rules }}$ and $\mathbf{C}_{c h}^{\text {usage }}$ take on binary and integer values, respectively, it is possible to use a standard mutation operator, which is employed in the classic genetic algorithm (Sivanandam and Deepa, 2008; Eiben and Smith, 2008). This type of mutation, in contrast to the mutations described in Section 4.3 , is not performed for each gene. The strength of the mutation results from the value of the parameter $p_{m} \in[0,1]$, which is called mutation probability (Sivanandam and Deepa, 2008; Eiben and Smith, 2008). The value of this parameter has to be set before the evolution process begins.

It should be noted that in our approach, during the evolution process, the chromosomes that encode systems useless from a practical point of view are removed. We assume that a useless system is the one with no inputs, no rules and/or no input fuzzy sets.

4.5. Chromosome evaluation. The evolutionary strategy that is used in a neuro-fuzzy system design 
process aims at minimizing the following fitness function for chromosome $\mathbf{C}_{c h}$ :

$$
\operatorname{Ff}\left(\mathbf{C}_{c h}\right)=\operatorname{Acc}\left(\mathbf{C}_{c h}\right)\left(1+\operatorname{Int}\left(\mathbf{C}_{c h}\right)\right),
$$

where

- $\operatorname{Acc}\left(\mathbf{C}_{c h}\right)$ determines the accuracy of the neuro-fuzzy system encoded in chromosome $\mathbf{C}_{c h}$ defined as a root mean square error (RMSE):

$$
\operatorname{Acc}\left(\mathbf{C}_{c h}\right)=\sqrt{\frac{\sum_{h=1}^{H} \sum_{j=1}^{m}\left(y_{h, j}-\hat{y}_{h, j}\right)^{2}}{m(H-1)}},
$$

where $m$ is the number of output signals, $H$ is the number of samples, $y_{h, j}$ is a value of the $j$-th output signal in the $h$-th sample determined by the model (7) and $y_{h, j}$ is a reference value of the $j$-th output signal in the $h$-th sample.

- The term $\operatorname{Int}\left(\mathbf{C}_{c h}\right)$ determines the degree of the fulfilment of the chosen interpretability criteria by the neuro-fuzzy system (13), encoded in the $\mathbf{C}_{c h}$ chromosome (18):

$$
\operatorname{Int}\left(\mathbf{C}_{c h}\right)=\frac{1}{4} \sum_{s=1}^{4} I_{s},
$$

where $I_{s}$ is the value of interpretability criteria defined by (14)- (17). The structure of Eqn. (29) allows for promotion of chromosomes that have a lower value of the component $\operatorname{Int}\left(\mathbf{C}_{c h}\right)\left(\operatorname{Int}\left(\mathbf{C}_{c h}\right) \in\right.$ $[0,1])$, i.e., that are distinguished by a more readable rule base. This is achieved by adding the value of 1 to the component $\operatorname{Int}\left(\mathbf{C}_{c h}\right)$.

\section{Simulations results}

During simulations we focused on two problems of nonlinear modelling:

1. a harmonic oscillator with variable pulsation,

2. a nonlinear electrical circuit (Jordan, 2006).

The values of the characteristic parameters of the evolutionary strategy common to all simulations are as follows: (a) the number of chromosomes in the parental population $\mu=50$, (b) the number of chromosomes in the temporary population $\lambda=200$, (c) constant $\epsilon_{0}=0.001$, (d) mutation probability $p_{m}=0.1$. The characteristic features and values of parameters of the neuro-fuzzy systems common to all simulations can be summarized as follows: (a) for input fuzzy sets we assumed the Gaussian membership function and for output singleton functions, (b) the maximum number of fuzzy sets for each input and output of the system and the maximum number of rules were set at $|\tilde{\mathcal{A}}|=|\mathcal{B}|=|\tilde{\mathcal{R}}|=9$. This number is taken into account in the paper concerning interpretability issues and it determines the maximum information which can be distinguished by a human directly. It is exactly $7 \pm 2$ and was established by Miller (1956). The threshold values of the constant used in the interpretability criteria (15)-(17) were set as follows: $\zeta=0.1,[\underline{\kappa}, \bar{\kappa}]=[0.2,0.6], \gamma=0.1$.

For both the problems, simulations were divided into two groups:

1. In the first case we focused on the accuracy of modelling. The purpose of the evolutionary strategy was to the select the parameters of the membership functions and the types of the inference operators ( $t$-norms). The interpretability component $\operatorname{Int}\left(\mathbf{C}_{c h}\right)$ of the fitness function (29) was not considered. The number of rules was set arbitrarily.

2. In the second case we focused on both the accuracy of modelling and the interpretability of the created neuro-fuzzy system. The purpose of the evolutionary strategy was to select parameters of membership functions, types of inference operators, and the number and forms of fuzzy rules. In the evaluation of chromosomes, the interpretability part of the fitness function was considered.

5.1. Problem of a harmonic oscillator with variable pulsation. The harmonic oscillator can be defined by the following equation (Ogata, 2004):

$$
\frac{\mathrm{d}^{2} x(t)}{\mathrm{d} t^{2}}+\omega^{2} x(t)=0
$$

where $\omega$ is an oscillator parameter. Taking $x_{1}(t)=\omega x(t)$ and $x_{2}(t)=\mathrm{d} x(t) / \mathrm{d} t$ as state variables, we obtain the following matrix representation of Eqn. (32):

$$
\left[\begin{array}{l}
\frac{\mathrm{d} x_{1}(t)}{\mathrm{d} t} \\
\frac{\mathrm{d} x_{2}(t)}{\mathrm{d} t}
\end{array}\right]=\left[\begin{array}{cc}
0 & \omega \\
-\omega & 0
\end{array}\right]\left[\begin{array}{l}
x_{1}(t) \\
x_{2}(t)
\end{array}\right] .
$$

In order to introduce nonlinearity to Eqns. 32 -33 we assume that parameter $\omega$ varies with the value of $x_{1}(t)$ according to the following equation:

$$
\omega\left(x_{1}\right)=2 \pi-\frac{\pi}{1+\left|2 x_{1}\right|^{6}} .
$$

Such a system reflects practical physical phenomena, e.g., a real electric generator with one of the elements (e.g., inductive) falling within the area of magnetic saturation above a certain current value.

In the simulations of this problem it is assumed that the system matrix $\mathbf{A}$ is given by the formula

$$
\mathbf{A}=\left[\begin{array}{cc}
0 & 2 \pi \\
-2 \pi & 0
\end{array}\right],
$$




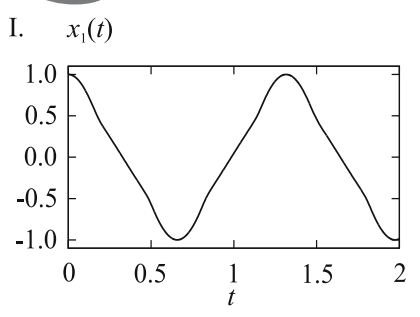

II. $x_{2}(t)$

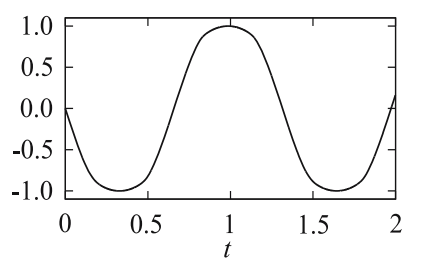

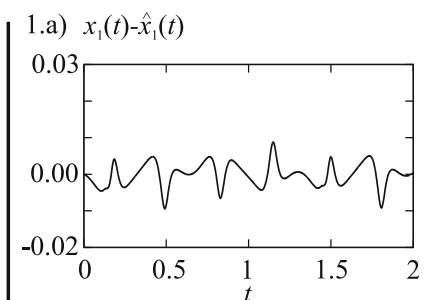

2.a) $x_{1}(t)-\hat{x}_{1}(t)$

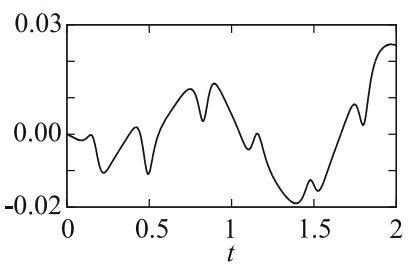

1.b) $x_{2}(t)-\hat{x}_{2}(t)$

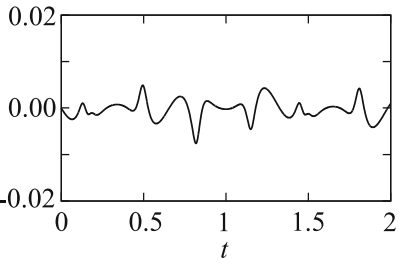

2.b) $x_{2}(t)-\hat{x}_{2}(t)$

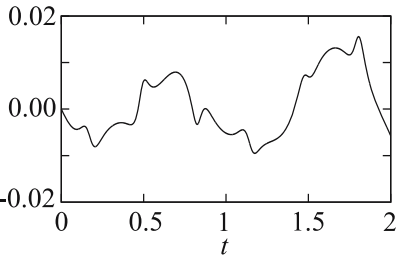

1.c) $\omega+p_{12}$

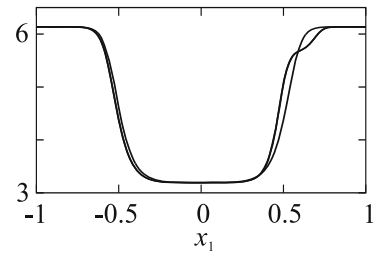

2.c) $\omega+p_{12}$

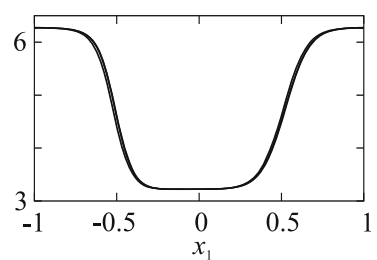

Fig. 3. Graphical illustration of the reference signals (I-II) and the results of modelling the harmonic oscillator by the fuzzy system 13] in the case of high accuracy (panels 1(a)-1(c)) and high interpretability (panels 2(a)-2(c)). Panels 1(a), 2(a) and 1(b), 2(b) show the error obtained for signals $x_{1}$ and $x_{2}$, respectively, panels 1(c) and 2(c) show the dependence of parameter $\omega+p_{12}$ of matrix $\mathbf{A}_{e q}$ on signal $x_{1}$. Panel 1(c) contains two curves because of the periodicity of the analysed function and because of the data set that was generated for $T=2 \mathrm{~s}$. The lines do not overlap because of the error obtained for signal $x_{1}$.

and the correction matrix is described as follows:

$$
\mathbf{P}_{\mathbf{A}}=\left[\begin{array}{cc}
0 & p_{12} \\
p_{21} & 0
\end{array}\right] \text {. }
$$

The simulations of oscillator were conducted for time interval $T=2 \mathrm{~s}$ with step $\mathrm{d} t=0.001 \mathrm{~s}$, so the training data set contains 2001 samples.

In the first group of simulations, whose purpose was to achieve the greatest accuracy of modelling, the best results were obtained for a fuzzy system composed of three rules and three fuzzy sets per each input and output of the system. It was noted that increasing this number does not have much effect on the value of the adjustment error RMSE (30). As a result of the evolutionary strategy, the obtained model can be summarized as follows:

- The accuracy of the model was RMSE $=0.0026$. The maximum absolute error for input $x_{1}$ was $\epsilon_{x_{1}}=$ 0.0094 and for input $x_{2}$ it was $\epsilon_{x_{2}}=0.0076$ (see Fig. 3). It follows that the prepared model well reproduces the actual signals.

- A detailed form of fuzzy rules of the the system (13) selected using the evolutionary strategy can be represented as follows

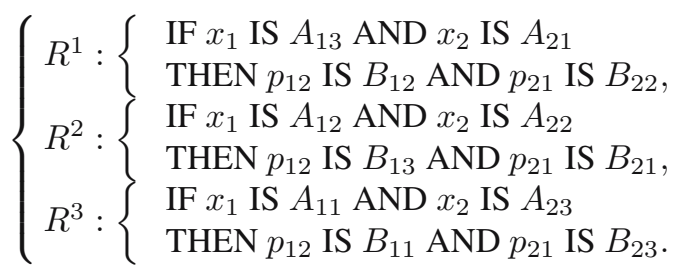

As explained in Section 2, the aim of the fuzzy system described by rules of the form (37) is to generate the values of coefficients of the matrix $\mathbf{P}_{\mathbf{A}}$. For this reason, in Eqn. (37) the system outputs are indicated as $p_{12}$ and $p_{21}$. The obtained membership functions are shown in Fig. 4(a) and the values of the interpretability criteria in Table 1 As can be seen, the obtained fuzzy sets overlap significantly. For this reason, it is difficult to associate a linguistic label with a clear interpretation, and the rules are difficult to read despite their small number.

For the second simulation conducted for this problem, aimed at gaining a system with the greatest accuracy while observing the conditions of interpretability, the obtained results can be summarized as follows:

- The accuracy of the model was RMSE $=0.0090$ and the maximum absolute error for input $x_{1}$ was $x_{1}=0.0246$ and for input $x_{2}$ it was $x_{2}=0.0155$. The obtained result is, as would be expected, worse than for the system described in the first variant (the system oriented on accuracy). However, it should be noted that the maximum absolute error was lower than $3 \%$ of the absolute value of the input signal, which can be considered a satisfactory result (see Fig. 3).

- A detailed form of fuzzy rules of the system (13) selected using the evolutionary strategy can be represented as follows: 
a)

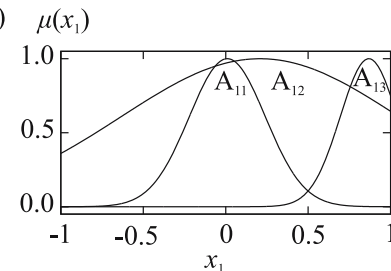

b) $\mu\left(x_{1}\right)$

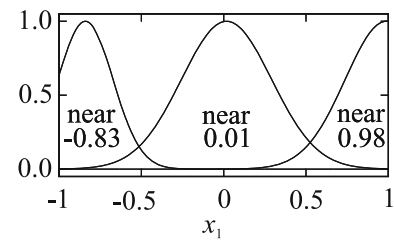

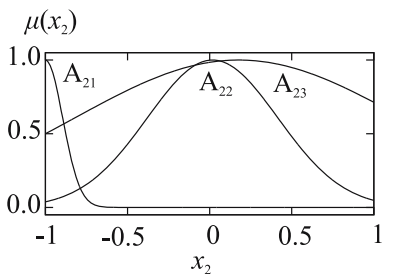

$\mu\left(p_{12}\right)$

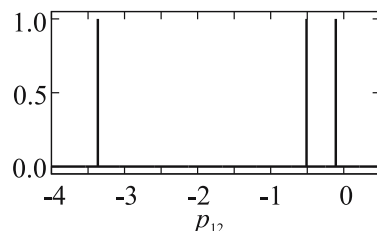

$\mu\left(p_{12}\right)$

input rejected by the evolutionary strategy

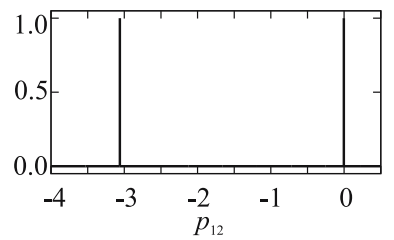

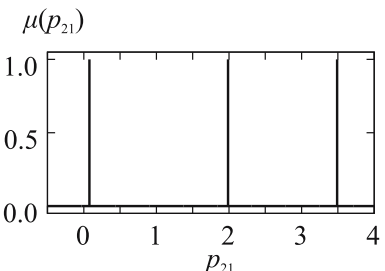

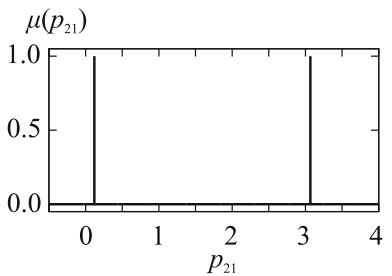

Fig. 4. Input and output fuzzy sets of the system used for modelling the harmonic oscillator in the case of high accuracy learning (a) as well as high interpretability and accuracy learning (b).

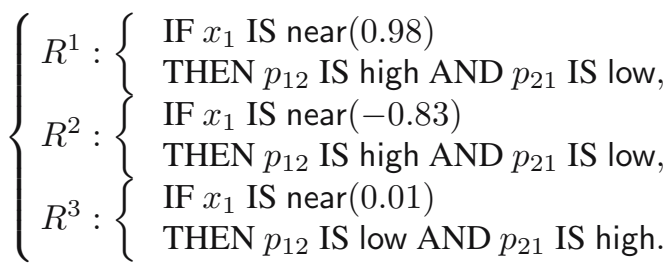

As in the case of the first simulation, the system was composed of three rules, but used only one input $x_{1}$. Additionally, the obtained membership functions were uniformly divided in the value spaces and overlapped each other to a much smaller degree, increasing the possibility of their interpretation (this is confirmed by the values obtained for each interpretability criterion presented in Table 1). The reduction of the complexity of the system and the ability to assign easily distinguishable linguistic labels made this system much easier to read than the one obtained in the previous simulation.

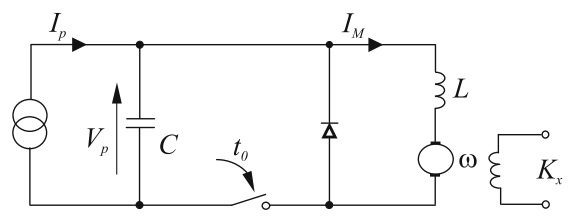

Fig. 5. Nonlinear electrical circuit with a solar generator and a DC drive system diagram.

5.2. Problem of a nonlinear electrical circuit. The second analysed problem concerns a nonlinear electrical circuit with a solar generator and a DC drive system (Jordan, 2006). The circuit is presented in Fig.5.
Table 1. Summary of the root mean square error 30 and the interpretability criteria (14)-177 for the problem of a harmonic oscillator. The boldface indicates a better result for each of the criteria considered.

\begin{tabular}{|c|c|c|c|}
\hline & \multicolumn{2}{|c|}{ Simulation variant } \\
\hline & & $\begin{array}{c}\text { high } \\
\text { accuracy }\end{array}$ & $\begin{array}{c}\text { high } \\
\text { interpretability }\end{array}$ \\
\hline \multicolumn{2}{|r|}{ RMSE } & 0.0026 & 0.0090 \\
\hline \multirow{5}{*}{ 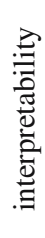 } & criterion 1 & 0.3330 & 0.2670 \\
\hline & criterion 2 & 0.0000 & 0.0000 \\
\hline & criterion 3 & 0.2096 & 0.0779 \\
\hline & criterion 4 & 0.3509 & 0.0000 \\
\hline & sum 31 & 0.2233 & 0.0862 \\
\hline
\end{tabular}

The nonlinear characteristics of the solar generator can be approximated using the following formula:

$$
I_{p}=I_{0}-I_{s}\left(e^{a V_{p}}-1\right),
$$

where $V_{p}$ is the solar generator voltage, $I_{0}$ is the photovoltaic current of the cell at $V_{p}=0$ (dependent on the light flux), $I_{s}$ is the saturation current defined by the Shockley equation and $a$ is the factor that characterizes the solar generator. This circuit can be described by the following system of nonlinear differential equations:

$$
\left\{\begin{array}{l}
\frac{\mathrm{d} u(t)}{\mathrm{d} t}=-\frac{I_{s}}{C} e^{a u(t)}-\frac{1}{C} i(t)+\frac{I_{s}+I_{0}}{C}, \\
\frac{\mathrm{d} i(t)}{\mathrm{d} t}=\frac{1}{L} i(t)-\frac{R_{m}}{L} u(t)-\frac{K_{x}}{L} \omega(t), \\
\frac{\mathrm{d} \omega(t)}{\mathrm{d} t}=\frac{K_{x}}{L} u(t)-\frac{K_{r}}{J} \omega(t),
\end{array}\right.
$$

where $u(t)$ is the generator voltage, $i(t)$ is the rotor 

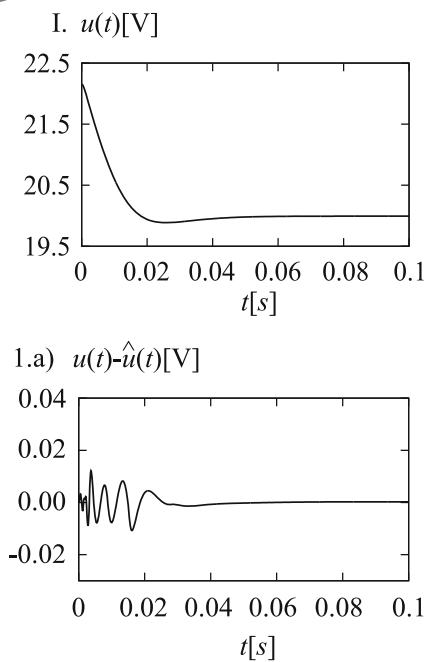

2.a) $u(t)-\hat{u}(t)[\mathrm{V}]$

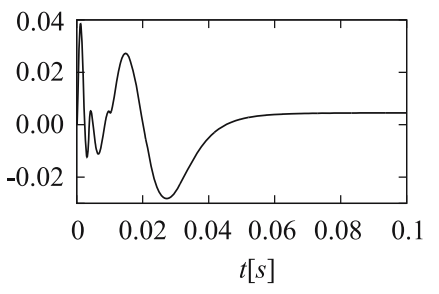

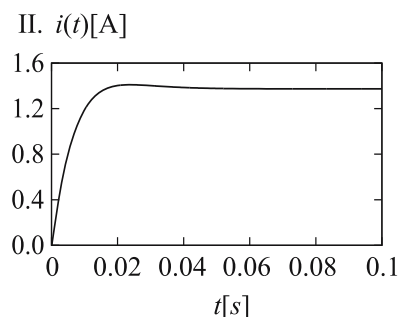

1.b) $i(t)-\hat{i}(t)[\mathrm{A}]$

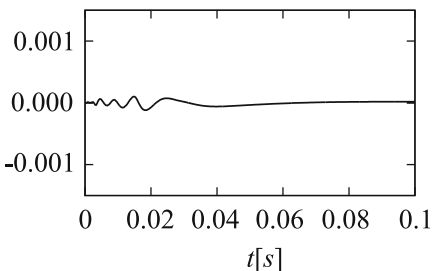

2.b) $i(t) \hat{i}(t)[\mathrm{A}]$

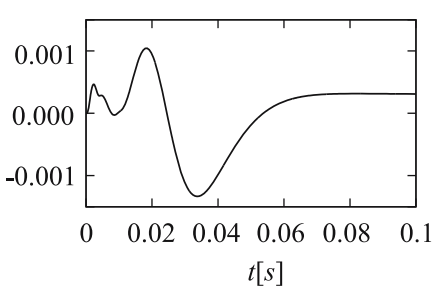

III. $\omega(t)[\mathrm{rad} / \mathrm{s}]$

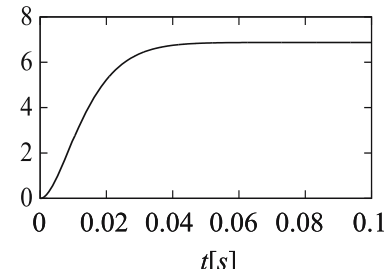

$t[s]$

1.c) $\omega(t)-\hat{\omega}(t)[\mathrm{rad} / \mathrm{s}]$

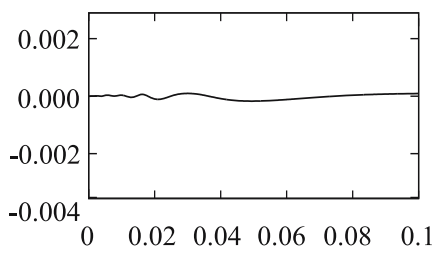

2.c) $\omega(t)-\hat{\omega}(t)[\mathrm{rad} / \mathrm{s}]$

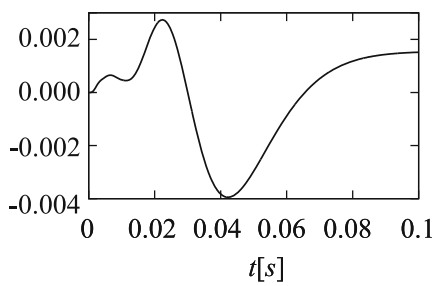

Fig. 6. Graphical illustration of the reference signals (I-III) and the results of modelling the nonlinear electrical circuit by the fuzzy system (13) in the case of high accuracy (panels 1(a)-1(c)) and high interpretability (panels 2(a)-2(c)) Panels 1(a) and 2(a) show the error obtained for signal $u, 1(\mathrm{~b})$ and 2(b) the error obtained for signal $i$, and 1(c) and 2(c) the error obtained for signal $\omega$.

current and $\omega(t)$ is the DC motor rotational speed. The parameters of circuit were set as follows: $R_{m}=$ $12.045 \Omega, C=500 \mu \mathrm{F}, L=0.1 \mathrm{H}, a=0.54 \mathrm{~V}^{-1}, K_{r}=$ $0.1 \mathrm{Vs}^{2}, I_{0}=2 \mathrm{~A}, J=10^{-3} \mathrm{Ws}^{3}, I_{s}=1.28$. $10^{-5} \mathrm{~A}, V_{p}=22.15 \mathrm{~V}, K_{x}=0.5 \mathrm{Vs}$. The values were taken from the work of Jordan (2006). The values of the system matrix were determined using Taylor's series expansion method at point $\mathbf{x}=[22.15,0.00,0.00]^{T}$ and are follows:

$$
\mathbf{A}=\left[\begin{array}{ccc}
-2163.86 & 2000.00 & 0.00 \\
10.00 & -120.45 & -5.00 \\
0.00 & 500.00 & -100.00
\end{array}\right]
$$

It should be noted that in the formulae (40) the nonlinearity occurs in the first of the equations in the part concerning the generator voltage. We assume that we know where the nonlinearity occurs but we do not know its characteristics, so the correction matrix is defined by the following formula:

$$
\mathbf{P}_{\mathbf{A}}=\left[\begin{array}{ccc}
p_{11} & 0 & 0 \\
0 & 0 & 0 \\
0 & 0 & 0
\end{array}\right]
$$

The results obtained in the first (focused on accuracy) simulation can be summarized as follows:
- The accuracy of the model was $R M S E=0.0026$. For signals $u, i$ and $\omega$, the maximum absolute errors were $\epsilon_{u}=0.0122 \mathrm{~V}, \epsilon_{i}=0.0001 \mathrm{~A}$ and $\epsilon_{\omega}=$ $0.0002 \mathrm{rad} / \mathrm{s}$, respectively (see Fig. 6). Thus, it can be concluded that the prepared model well reproduces the actual signals.

- A detailed form of fuzzy rules of the system (13) selected by the evolutionary strategy can be presented as

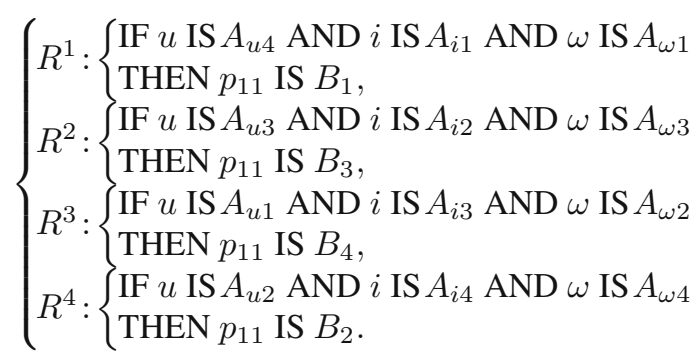

The shapes of membership functions are presented in Fig. 7 (a). As in the problem considered previously, the obtained functions highly overlap, making it 
difficult to interpret and impossible to associate clear linguistic labels with fuzzy sets. Thus, the fuzzy rules (43) are difficult to read. The values of each interpretability criterion given by 114 - 17) are presented in Table 2 This justifies the need to include these criteria in the fuzzy system design process.

In the second group of simulations conducted for the problem considered, the best obtained model can be characterized by the following properties:

- The accuracy of the model was RMSE $=0.0083$. For signals $u, i$ and $\omega$, the maximum absolute errors were $\epsilon_{u}=0.0384 \mathrm{~V}, \epsilon_{i}=0.0013 \mathrm{~A}$ and $\epsilon_{\omega}=0.0039 \mathrm{rad} / \mathrm{s}$, respectively (see Fig. 6). From this it can be concluded that the error rate slightly increased; however, the resulting accuracy is still at a satisfactory level.

- A detailed form of fuzzy rules of the system (13) selected by the evolutionary strategy can be presented as

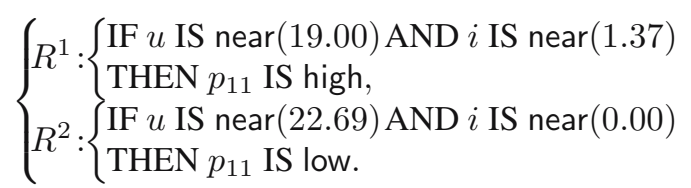

The shapes of membership functions are presented in Fig. 7(b). Those functions are characterized by a high degree of interpretability (as confirmed by the values obtained for the different criteria listed in Table 2), allowing us to assign clear linguistic labels to these functions. In addition, it should be noted that during the evolutionary process the created fuzzy system consisted only of two rules and used two inputs. This resulted in a significant decrease in the system complexity and a further increase in its readability.

\section{Conclusions}

In this paper a new method of modelling a nonlinear dynamic system was presented. It is based on combining the state-space modelling approach with methods of computational intelligence. In particular, it uses the possibilities of neuro-fuzzy systems to generate the values of the correction matrix, at each new operating point, which are added to the system matrix. This solution enables more accurate modelling of systems in those areas where their characteristics are nonlinear. To determine the system structure and the parameters of membership functions, the $(\lambda+\mu)$ evolutionary strategy was used. An important element of the proposed method is taking into account different interpretability criteria
Table 2. Summary of the root mean square error 30 and the interpretability criteria 14- 17) for the problem of a nonlinear electrical circuit. The bold type indicates a better result for each of the criteria considered.

\begin{tabular}{|c|c|c|c|}
\hline & \multicolumn{2}{|c|}{ Simulation variant } \\
\hline & & $\begin{array}{c}\text { high } \\
\text { accuracy }\end{array}$ & $\begin{array}{c}\text { high } \\
\text { interpretability }\end{array}$ \\
\hline \multicolumn{2}{|r|}{ RMSE } & 0.0026 & 0.0083 \\
\hline \multirow{5}{*}{ 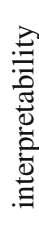 } & criterion 1 & 0.4440 & 0.1780 \\
\hline & criterion 2 & 0.0000 & 0.0000 \\
\hline & criterion 3 & 0.2099 & 0.0000 \\
\hline & criterion 4 & 0.4693 & 0.0305 \\
\hline & sum5 31 & 0.2808 & 0.0520 \\
\hline
\end{tabular}

in the fuzzy system design process. This resulted in systems with lower complexity, greater readability of fuzzy rules included in the rule base, and operating with acceptable accuracy. This also makes it possible to assign specific linguistic labels to fuzzy sets. The effectiveness of the proposed method was confirmed by the performed simulations.

\section{Acknowledgment}

The authors would like to thank the reviewers for very helpful suggestions and comments in the revision process.

The project was financed by the Polish National Science Center on the basis of the decision DEC-2012/05/B/ST7/02138.

\section{References}

Adjrad, M. and Belouchrani, A. (2007). Estimation of multicomponent polynomial-phase signals impinging on a multisensor array using state-space modeling, IEEE Transactions on Signal Processing 55(1): 32-45.

Angelov, P.P., Filev, D.P. (2004). Flexible models with evolving structure, International Journal of Intelligent Systems 19(4): 327-340.

Babuska, R., Verbruggen, H. (2003). Flexible neuro-fuzzy methods for nonlinear system identification, Annual Reviews in Control 27(1): 73-85.

Bagarinao, E., Matsuo, K., Nakai, T. and Sato, S. (2003). Estimation of general linear model coefficients for real-time application, NeuroImage 19(2): 422-429.

Banerjee, A., Arkun, Y., Ogunnaike, B. and Pearson, R. (1997). Estimation of nonlinear systems using linear multiple models, AIChE Journal 43(5): 1204-1226.

Bohlin, T.P. (2006). Practical Grey-Box Process Identification: Theory and Applications, Springer, London.

Boukezzoula, R., Galichet, S. and Foulloy, L. (2007). Fuzzy feedback linearizing controller and its equivalence with the 
a) $\mu(\mathrm{u})$

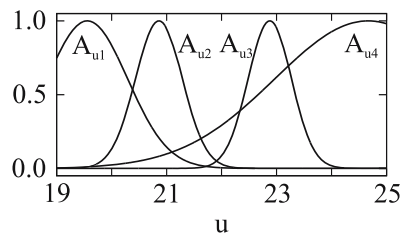

b) $\mu(\mathrm{u})$

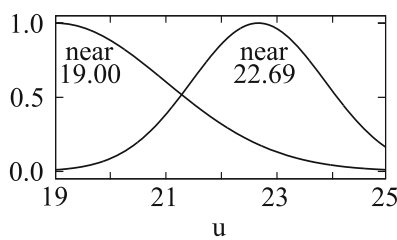

$\mu(\mathrm{i})$

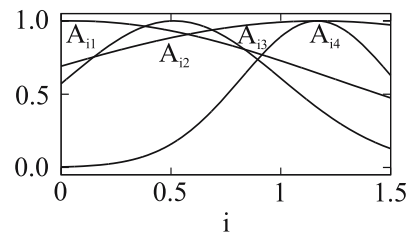

$\mu(\mathrm{i})$

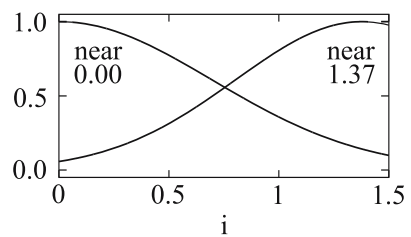

$\mu(\omega)$

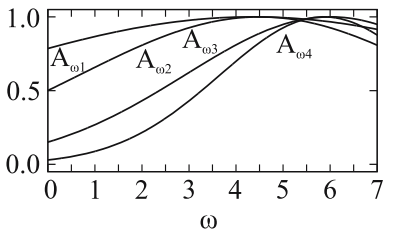

input rejected by the evolutionary strategy $\mu\left(p_{11}\right)$

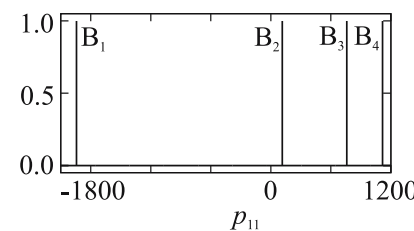

$\mu\left(p_{11}\right)$

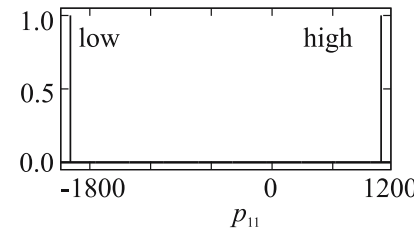

Fig. 7. Graphical illustration of fuzzy sets used in the fuzzy system 13 which is employed for modelling the nonlinear electrical circuit in the case of high accuracy (a) and high interpretability (b). Since in the second case the input $\omega$ is not used, we do not present its fuzzy partition.

fuzzy nonlinear internal model control structure, International Journal of Applied Mathematics and Computer Science 17(2): 233-248, DOI: 10.2478/v10006-007-0021-4.

Casillas, J., Cordón, O., Herrera, F. and Magdalena, L. (2003). Interpretability improvements to find the balance interpretability-accuracy in fuzzy modeling: An overview, in J. Casillas et al. (Eds.), Interpretability Issues in Fuzzy Modeling, Springer, Berlin/Heidelberg, pp. 3-22.

Caughey, T.K. (1963). Equivalent linearization techniques, Journal of the Acoustical Society of America 35(11): 1706-1711.

Cordón, O. (2011). A historical review of evolutionary learning methods for Mamdani-type fuzzy rule-based systems: Designing interpretable genetic fuzzy systems, International Journal of Approximate Reasoning 52(6): 894-913.

Cordón, O., Herrera, F., Hoffmann, F. and Magdalena, L. (2001). Genetic Fuzzy Systems, World Scientific Publishing Company, Singapore.

Cpałka, K. (2009a). A new method for design and reduction of neuro-fuzzy classification systems, IEEE Transactions on Neural Networks 20(4): 701-714.

Cpałka, K. (2009b). On evolutionary designing and learning of flexible neuro-fuzzy structures for nonlinear classification, Nonlinear Analysis A: Theory, Methods and Applications 71(12): 1659-1672.

Cpałka, K., Łapa, K., Przybył, A. and Zalasiński, M. (2014). A new method for designing neuro-fuzzy systems for nonlinear modelling with interpretability aspects, Neurocomputing 135: 203-217.

Cpałka, K., Rebrova, O., Nowicki, R. and Rutkowski, L. (2013). On design of flexible neuro-fuzzy systems for nonlinear modelling, International Journal of General Systems 42(6): 706-720.

Czekalski, P. (2006). Evolution-fuzzy rule based system with parameterized consequences, International Journal of Applied Mathematics and Computer Science 16(3): 373-385.
DeHaan, D. and Guay, M. (2006). A new real-time perspective on non-linear model predictive control, Journal of Process Control 16(6): 615-624.

Di Nuovo, A. and Ascia, G. (2013). A fuzzy system index to preserve interpretability in deep tuning of fuzzy rule based classifiers, Journal of Intelligent and Fuzzy Systems 25(2): 493-504.

Eiben, A.E. and Smith, J. (2008). Introduction to Evolutionary Computing, Springer, Berlin/Heidelberg.

Fei, X., Lu, C.-C. and Liu, K. (2011). A Bayesian dynamic linear model approach for real-time short-term freeway travel time prediction, Transportation Research C: Emerging Technologies 19(6): 1306-1318.

Fogel, D.B. (2006). Evolutionary Computation: Toward a New Philosophy of Machine Intelligence, Vol. 1, John Wiley \& Sons, Hoboken, NJ.

Fogel, D.B. and Atmar, J.W. (1990). Comparing genetic operators with Gaussian mutations in simulated evolutionary processes using linear systems, Biological Cybernetics 63(2): 111-114.

Forst, W. and Hoffmann, D. (2010). Optimization Theory and Practice, Springer, New York, NY.

Gabryel, M. and Rutkowski, L. (2006). Evolutionary learning of Mamdani-type neuro-fuzzy systems, in L. Rutkowski et al. (Eds.), Artificial Intelligence and Soft Computing, Lecture Notes in Computer Science, Vol. 4029, Springer, Berlin/Heidelberg, pp. 354-359.

Gacto, M., Alcala, R. and Herrera, F. (2011). Interpretability of linguistic fuzzy rule-based systems: An overview of interpretability measures, Information Sciences 181(20): $4340-4360$.

Grabowski, P. and Callier, F.M. (2001). Circle criterion and boundary control systems in factor form: Input-output approach, Applied Mathematics and Computer Science 11(6): 1387-1403. 
Háber, R. and Keviczky, L. (1999). Nonlinear System Identification-Input-Output Modeling Approach, Vol. 1: Nonlinear System Parameter Identification, Springer Netherlands, Dordrecht.

Homaifar, A. and McCormick, E. (1995). Simultaneous design of membership functions and rule sets for fuzzy controllers using genetic algorithms, IEEE Transactions on Fuzzy Systems 3(2): 129-139.

Horzyk, A. and Tadeusiewicz, R. (2004). Self-optimizing neural networks, Advances in Neural Networks, Springer, Berlin/Heidelberg, pp. 150-155.

Huijberts, H., Nijmeijer, H. and Willems, R. (2000). System identification in communication with chaotic systems, IEEE Transactions on Circuits and Systems I: Fundamental Theory and Applications 47(6): 800-808.

Ikonen, E. and Najim, K. (2001). Advanced Process Identification and Control, Vol. 9, CRC Press, New York, NY.

Ishibashi, R. and Lucio Nascimento, Jr., C. (2013). GFRBS-PHM: A genetic fuzzy rule-based system for PHM with improved interpretability, IEEE Conference on Prognostics and Health Management, 2013, Gaithersburg, MD, USA, pp. 1-7.

Ishibuchi, H. and Yamamoto, T. (2004). Fuzzy rule selection by multi-objective genetic local search algorithms and rule evaluation measures in data mining, Fuzzy Sets and Systems 141(1): 59-88.

Jang, I.-S. R. and Sun, C.-T. (1995). Neuro-fuzzy modeling and control, Proceedings of the IEEE 83(3): 378-406.

Johansen, T.A., Shorten, R. and Murray-Smith, R. (2000). On the interpretation and identification of dynamic Takagi-Sugeno fuzzy models, IEEE Transactions on Fuzzy Systems 8(3): 297-313.

Johansson, U., Sönströd, C., Norinder, U. and Boström, H. (2011). Trade-off between accuracy and interpretability for predictive in silico modeling, Future Medicinal Chemistry 3(6): 647-663.

Jordan, A. (2006). Linearization of non-linear state equation, Bulletin of the Polish Academy of Sciences: Technical Sciences 54(1): 63-73.

Juang, C.-F. and Chen, C.-Y. (2013). Data-driven interval type-2 neural fuzzy system with high learning accuracy and improved model interpretability, IEEE Transactions on Cybernetics 43(6): 1781-1795.

Kim, M.-S., Kim, C.-H. and Lee, J.-J. (2006). Evolving compact and interpretable Takagi-Sugeno fuzzy models with a new encoding scheme, IEEE Transactions on Systems, Man, and Cybernetics B: Cybernetics 36(5): 1006-1023.

Kluska, J. (2009). Analytical Methods in Fuzzy Modeling and Control, Springer, Berlin/Heidelberg.

Kluska, J. (2015). Selected applications of P1-TS fuzzy rule-based systems, in L. Rutkowski et al. (Eds.), Artificial Intelligence and Soft Computing, Lecture Notes in Computer Science, Vol. 9119, Springer, Berlin/Heidelberg, pp. 195-206.
Kristensen, N.R., Madsen, H. and Jørgensen, S.B. (2004) A method for systematic improvement of stochastic grey-box models, Computers \& Chemical Engineering 28(8): 1431-1449.

Kroese, D.P., Taimre, T. and Botev, Z.I. (2011). Handbook of Monte Carlo Methods, Vol. 706, John Wiley \& Sons, Hoboken, NJ.

Li, C. and Chiang, T.-W. (2012). Intelligent financial time series forecasting: A complex neuro-fuzzy approach with multi-swarm intelligence, International Journal of Applied Mathematics and Computer Science 22(4): 787-800, DOI: 10.2478/v10006-012-0058-x.

Ljung, L. (2010). Approaches to identification of nonlinear systems, 9th Chinese Control Conference, Beijing, China, pp. $1-5$.

Łęski, J. (2003). A fuzzy if-then rule-based nonlinear classifier, International Journal of Applied Mathematics and Computer Science 13(2): 215-223.

Lughofer, E. (2013). On-line assurance of interpretability criteria in evolving fuzzy systems-achievements, new concepts and open issues, Information Sciences 251: $22-46$.

Malchiodi, D. and Pedrycz, W. (2013). Learning membership functions for fuzzy sets through modified support vector clustering, in F. Masulli et al. (Eds.), Fuzzy Logic and Applications, Springer, Cham, pp. 52-59.

Medasani, S., Kim, J. and Krishnapuram, R. (1998). An overview of membership function generation techniques for pattern recognition, International Journal of Approximate Reasoning 19(3): 391-417.

Miller, G.A. (1956 ). The magical number seven, plus or minus two: Some limits on our capacity for processing information, The Psychological Review 63: 81-97.

Mrugalski, M. (2014). Advanced Neural Network-Based Computational Schemes for Robust Fault Diagnosis, Studies in Computational Intelligence, Vol. 510, Springer-Verlag, Berlin/Heidelberg.

Murray-Smith, R. and Johansen, T. (1997). Multiple Model Approaches to Nonlinear Modelling and Control, CRC Press, Boca Raton, FL.

Nelles, O. (2001). Nonlinear System Identification: From Classical Approaches to Neural Networks and Fuzzy Models, Springer, Berlin/Heidelberg.

Ogata, K. (2004). System Dynamics, Pearson/Prentice Hall, Upper Saddle River, NJ.

Patton, R.J., Korbicz, J., Witczak, M. and Uppal, F. (2005). Combined computational intelligence and analytical methods in fault diagnosis, IEE Control Engineering Series 70: 349.

Pedro, J.O. and Dahunsi, O.A. (2011). Neural network based feedback linearization control of a servo-hydraulic vehicle suspension system, International Journal of Applied Mathematics and Computer Science 21(1): 137-147, DOI: 10.2478/v10006-011-0010-5. 
Przybył, A. and Jelonkiewicz, J. (2003). Genetic algorithm for observer parameters tuning in sensorless induction motor drive, Proceedings of the 6th International Conference on Neural Networks and Soft Computing, Zakopane Poland, pp. 376-381.

Puig, V., Witczak, M., Nejjari, F., Quevedo, J. and Korbicz, J. (2007). A GMDH neural network-based approach to passive robust fault detection using a constraint satisfaction backward test, Engineering Applications of Artificial Intelligence 20(7): 886-897.

Quah, K.H. and Quek, C., (2006). FITSK: Online local learning with generic fuzzy input Takagi-Sugeno-Kang fuzzy framework for nonlinear system estimation, IEEE Transactions on Systems, Man, and Cybernetics B: Cybernetics 36(1): 166-178.

Roffel, B. and Betlem, B.H. (2004). Advanced Practical Process Control, Springer, Berlin/Heidelberg.

Rüping, S. (2006). Learning Interpretable Models, Ph.D. thesis, Technical University of Dortmund, Dortmund.

Rutkowski, L. (2008). Computational Intelligence: Methods and Techniques, Springer, Berlin/Heidelberg.

Rutkowski, L. and Cpałka, K. (2005). Designing and learning of adjustable quasi-triangular norms with applications to neuro-fuzzy systems, IEEE Transactions on Fuzzy Systems 13(1): 140-151.

Salapa, K., Trawińska, A., Roterman, I. and Tadeusiewicz, R. (2014). Speaker identification based on artificial neural networks. Case study: The Polish vowel (a pilot study), Bio-Algorithms and Med-Systems 10(2): 91-99.

Setnes, M. and Roubos, H. (2000). GA-fuzzy modeling and classification: Complexity and performance, IEEE Transactions on Fuzzy Systems 8(5): 509-522.

Schröder, D. (Ed.) (2000). Intelligent Observer and Control Design for Nonlinear Systems, Springer, Berlin/Heidelberg.

Shill, P., Akhand, M. and Murase, K. (2011). Simultaneous design of membership functions and rule sets for type-2 fuzzy controllers using genetic algorithms, 14th International Conference on Computer and Information Technology, Dhaka, Bangladesh, pp. 554-559.

Shukla, P. and Tripathi, S. (2013). Interpretability issues in evolutionary multi-objective fuzzy knowledge base systems, 7th International Conference on Bio-Inspired Computing: Theories and Applications, Madhya Pradesh, India, pp. 473-484.

Sivanandam, S. and Deepa, S. (2008). Genetic Algorithm Optimization Problems, Springer, Berlin/Heidelberg.

Starczewski, J.T., Bartczuk, Ł., Dziwiński, P. and Marvuglia, A. (2010). Learning methods for type-2 FLS based on FCM, in L. Rutkowski et al. (Eds.), Artificial Intelligence and Soft Computing, Springer, Berlin/Heidelberg, pp. 224-231.

Tadeusiewicz, R. (2010). Using neural networks for simplified discovery of some psychological phenomena, in L. Rutkowski et al. (Eds.), Artificial Intelligence and Soft Computing, Springer, Berlin/Heidelberg, pp. 104-123.
Tadeusiewicz, R., Chaki, R. and Chaki, N. (2014). Exploring Neural Networks with C\#, CRC Press, Boca Raton, FL.

Tadeusiewicz, R. and Figura, I. (2011). Phenomenon of tolerance to damage in artificial neural networks, Computer Methods in Materials Science 11(4): 501-513.

Tan, Y. (2004). Time-varying time-delay estimation for nonlinear systems using neural networks, International Journal of Applied Mathematics and Computer Science 14(1): 63-68.

Wang, H., Kwong, S., Jin, Y., Wei, W. and Man, K.-F. (2005). Agent-based evolutionary approach for interpretable rule-based knowledge extraction, IEEE Transactions on Systems, Man, and Cybernetics C: Applications and Reviews 35(2): 143-155.

Wang, H., Kwong, S., Jin, Y., Wei, W. and Man, K.-F. (2005). Multi-objective hierarchical genetic algorithm for interpretable fuzzy rule-based knowledge extraction, Fuzzy Sets and Systems 149(1): 149-186.

Wilamowski, B.M. (2005). Methods of computational intelligence for nonlinear control systems, ICCAE 2005 International Conference on Control, Automation and System, Gyeonggi-Do, Korea, pp. P1-P8.

Witkowska, A. and Śmierzchalski, R. (2012). Designing a ship course controller by applying the adaptive backstepping method, International Journal of Applied Mathematics and Computer Science 22(4): 985-997, DOI: 10.2478/v10006-012-0073-y.

Wu, C.-J. and Liu, G.-Y. (2000). A genetic approach for simultaneous design of membership functions and fuzzy control rules, Journal of Intelligent and Robotic Systems 28(3): 195-211.

Xie, Y., Guo, B., Xu, L., Li, J. and Stoica, P. (2006) Multistatic adaptive microwave imaging for early breast cancer detection, IEEE Transactions on Biomedical Engineering 53(8): 1647.

Zhou, S.-M., Gan, J. Q. (2008). Low-level interpretability and high-level interpretability: A unified view of data-driven interpretable fuzzy system modelling, Fuzzy Sets and Systems 159(23): 3091-3131.

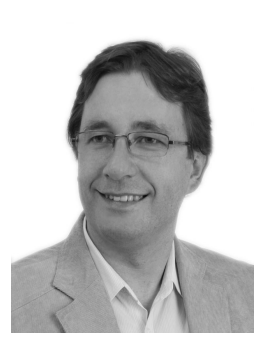

Eukasz Bartczuk was born in Częstochowa, Poland, in 1979. He received the M.Sc. and $\mathrm{Ph} . \mathrm{D}$. degrees in computer science in 2003 and 2008, respectively, both from the Częstochowa University of Technology. Since 2008 he has been an assistant professor at the Institute of Computational Intelligence there. He has published about 15 technical papers in conference proceedings and book chapters. His main fields of interests include fuzzy systems, evolutionary algorithms, and hybrid methods. 


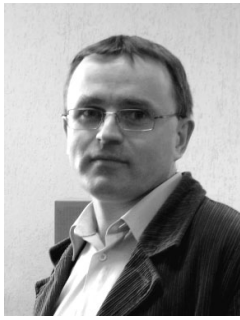

Andrzej Przybył was born in Brzeziny, Poland, in 1972. He received the Ph.D. degree in automatic control and robotics from the Poznań University of Technology, Poland, in 2003. He is an assistant professor at the Institute of Computational Intelligence, Częstochowa University of Technology, Poland. He works on developing new control methods used in mechatronic systems. His research interests center around motion control systems, real-time Ethernet, fieldprogrammable gate array (FPGA) devices, and soft computing algorithms for electrical drives. He has designed various microprocessors, digital signal processors, and FPGA-based embedded systems. He has published about 40 technical papers.

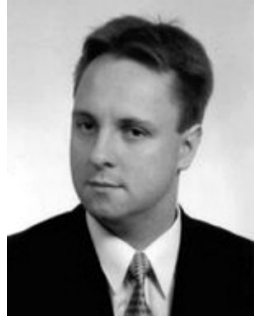

Krzysztof Cpałka was born in Częstochowa, Poland, in 1972. He received the M.Sc. degree in electrical engineering in 1997 and the $\mathrm{Ph} . \mathrm{D}$. degree (with honors) in computer engineering in 2002, both from the Częstochowa University of Technology, Poland, and the D.Sc. degree in computer engineering from the Systems Research Institute of the Polish Academy of Sciences in 2010. Since 2010, he has been an associate professor in the Institute of Computational Intelligence at the Częstochowa University of Technology. He has published over 80 technical papers in journals, conference proceedings, and book chapters. His research interests include fuzzy systems, evolutionary algorithms, and hybrid methods.
Received: 27 June 2015

Revised: 21 January 2016

Re-revised: 20 May 2016

Accepted: 15 June 2016 